\title{
Multiscale models of colloidal dispersion of particles in nematic liquid crystals
}

\author{
T. P. Bennett, ${ }^{1}$ G. D'Alessandro, ${ }^{1}$ and K. R. Daly ${ }^{2}$ \\ ${ }^{1}$ Mathematical Sciences, University of Southampton, Southampton, England, United Kingdom \\ ${ }^{2}$ Engineering Sciences, University of Southampton, Southampton, England, United Kingdom \\ (Received 13 June 2014; revised manuscript received 16 September 2014; published 15 December 2014)
}

\begin{abstract}
We use homogenization theory to develop a multiscale model of colloidal dispersion of particles in nematic liquid crystals under weak-anchoring conditions. We validate the model by comparing it with simulations by using the Landau-de Gennes free energy and show that the agreement is excellent. We then use the multiscale model to study the effect that particle anisotropy has on the liquid crystal: spherically symmetric particles always reduce the effective elastic constant. Asymmetric particles introduce an effective alignment field that can increase the Fredericks threshold and decrease the switch-off time.
\end{abstract}

DOI: 10.1103/PhysRevE.90.062505

PACS number(s): 61.30.Dk, 61.30.Gd, 61.30.Hn

\section{INTRODUCTION}

There is considerable research interest in colloidal, or doped, nematic liquid crystals. These consist of micronto nanometer-sized particles suspended in a nematic host. Dopants may posses permanent electric or magnetic dipoles leading to ferronematic systems, or their influence may be due to mechanical anchoring on the dopant surface. Doped nematics can be loosely categorized as being in the strongor weak-anchoring limit by the size of the dopants and the anchoring strength.

Systems dominated by the anchoring at the surface of dopants have been investigated experimentally [1,2], geometrically [3], and numerically [4]. In these systems complex defect structures encircling dopant particles can form. In the stronganchoring limit point and line defects and their accompanying elastic distortions introduce long-range correlations between the dopant particles [4]. Numerical studies have shown that spherical colloids can aggregate into two-dimensional crystals bound together by topological defects [5]. The stability of a particular defect configuration depends not only on the particle size but on the anchoring strength and applied field [6]. Conversely, in the weak-anchoring limit, defects are not induced and elastic deformations are small, even though aggregation still occurs on a long enough timescale [7]. In general, stiffening the liquid crystals, weakening the anchoring, and reducing particle size all move a system towards the weak-anchoring limit [7].

In this paper we focus on the weak-anchoring case, i.e., the liquid crystal alignment is dominated by elastic and electrostatic forces with the dopants acting to alter rather than dominate the properties of the liquid crystal. Their effect, however, may still be significant. For example, gold nanoparticle inclusions [8] have a considerable effect on the liquid crystal hosts: they lower the nematic-isotropic transition temperature [9], increase the switching speed [10], and introduce tunability of the dielectric anisotropy [11]. More recently, gold particles $[12,13]$ and layered structures [14] have been studied for their applications to the creation of tunable metamaterials.

Ferromagnetic and ferroelectric particles are other classes of dopants that have attracted significant interest because of their potential to improve the properties of liquid crystals. Interest in ferronematic systems was initially raised by de Gennes [15]: the addition of dopants alters the response of the nematic host to external fields due to the coupling between the dopants dipole and applied field. Because the orientation of the dopants is correlated with the director, the orienting effect of the applied field on the dopants is felt by the nematic. Current theories on liquid crystals doped with ferromagnetic [16-20] or ferroelectric [21,22] particles assume a mean field interaction between the particles and the director field.

Effective medium theories for doped liquid crystals are based on additional terms added to the free energy of the liquid crystal. These terms characterize the reorientation of the dopant particles in addition to their interaction with the liquid crystal and external fields. While the dielectric response of the liquid crystal is altered by these terms, in general these theories neglect the influence of the dopant on the elastic properties of the nematic: the anchoring is assumed to align the dopant with the nematic without causing additional stiffness or memory effects in the nematic. More importantly, phenomenological parameters are required to describe the coupling between dopants dipole and external fields. These parameters have been fitted with some success using experimental data [23], but there is clearly a need for a macroscopic model whose parameters are derived self-consistently from the microscopic equations.

This paper outlines the first steps in developing such a theory. This theory aims to produce a macroscopic model for colloidal nematics that explicitly treats the microscopic physics and geometry of the colloidal particles. We consider a liquid crystal confined by a set of evenly distributed inclusions of fixed orientation and position. The inclusions we consider may be identified with a periodic microstructure or with fixed dopant particles. We discuss in Sec. IV the consequences of this approximation and how it may be relaxed in future work. The size and volume fraction of the particles are assumed small with respect to the macroscopic size of the device. Hence, the spacing between these inclusions is small with respect to the length scale of interest, but not so small that a $\mathcal{Q}$-tensor macroscopic theory is no longer valid (e.g., 30 to $50 \mathrm{~nm}$ with respect to micron-sized macroscopic lengths). This length scale ratio forms the basis of the homogenization technique and allows us to derive averaged or "homogenized" equations which capture the underlying geometrical effects of the inclusions through a representative cell problem [24]. The cell problem allows us to relate the macroscopic parameters to the microstructure of the particle-liquid-crystal interaction. 
This approach enables us to form a direct link between the geometry of the microstructure or dopant distribution and the effective material parameters used to describe the macroscopic system.

Existing literature relating to homogenization and liquid crystals [25-27] prove under general conditions the convergence of the homogenization scheme based on the director representation of the nematic liquid crystal. In particular, they assume that the anchoring is characterized by a preferred direction on the dopant surface and a single anchoring energy. Here we consider a similar system, but derive the multiscale equations using the $Q$-tensor formalism. Our aim is to verify them against numerical simulations and to use them to study the effect of particle anisotropy on the liquid crystal.

The multiscale approach offered by homogenization theory has many advantages with respect to a full $Q$-tensor simulation. First, it clearly identifies the physical effects of the inclusion of particles on the liquid crystal, in terms of modification of the macroscopic parameters of a pure liquid crystal, or the creation of new terms and effective fields. Second, it allows these corrections and the resulting effective fields to be computed directly from a microscopic theory. While these computations may be complex and costly, they are required only once for identical inclusions. Finally, the complex geometry resulting from the inclusion of particles severely increases the computation time of a $Q$-tensor simulation. The homogenized equations do not suffer from this effect, because their geometry is the same as that of an undoped system.

This paper is organized as follows: in Sec. II we develop the multiscale model, present the main results of this paper, and outline the assumptions we made in modeling our system. In Sec. III we validate the model by comparing numerical simulations of the full system with the predictions of the homogenized equations. We then use the homogenized model to study the effect of the particle inclusion on the Fredericks transition and on the switch-on and -off time of a planar cell. In particular, we show that by using ellipsoidal inclusions the switch-on or switch-off time can be reduced. Finally, in Sec. IV we discuss the approximations at the base of the homogenized model and how they may be relaxed.

\section{THEORY}

\section{A. Governing equations}

We describe the alignment of the nematic by the $\mathcal{Q}$ tensor [28] which, for a general biaxial nematic liquid crystal, is given by

$$
\tilde{\mathcal{Q}}=\sqrt{\frac{3}{2}} \tilde{S}\left(\hat{\boldsymbol{d}} \otimes \hat{\boldsymbol{d}}-\frac{\mathcal{I}}{3}\right)+\sqrt{\frac{3}{2}} \tilde{\beta}\left(\hat{\boldsymbol{m}} \otimes \hat{\boldsymbol{m}}-\frac{\mathcal{I}}{3}\right) .
$$

Here $\tilde{S}$ is the scalar order parameter and $\tilde{\beta}$ is the biaxiality parameter, $\mathcal{I}$ is the identity matrix, $\hat{\boldsymbol{d}}$ is the liquid crystal director field, and $\hat{\boldsymbol{m}}$ is a unit vector defining the second principal axis of $\tilde{\mathcal{Q}}$, which is orthogonal to the director. We describe the liquid crystal orientation through a free energy minimization.

In constructing our model we make several assumptions about our system. In order to apply homogenization we assume periodicity, i.e., the inclusions form a periodic array of unit

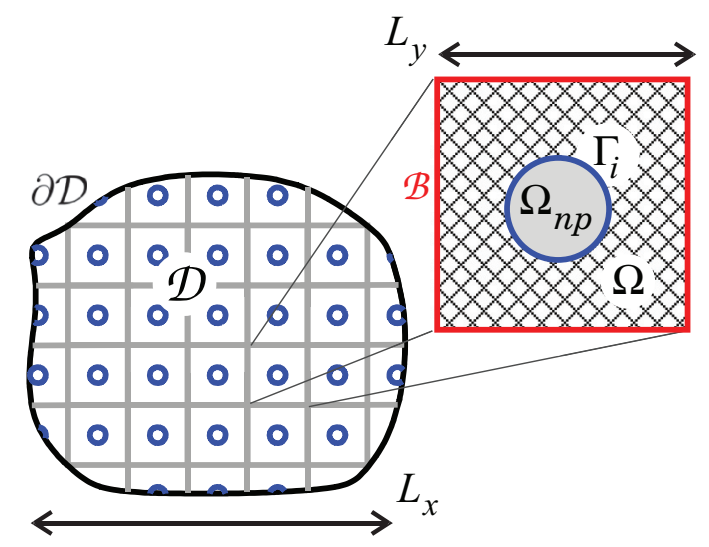

FIG. 1. (Color online) Domains used in defining scale separation. The macroscopic domain consists of an open region $\mathcal{D}$, with external boundary $\partial \mathcal{D}$, constructed from unit cells $\Omega$ with outer boundaries $\mathcal{B}$ and metallic inclusions of volume $\Omega_{n p}$ and boundary $\Gamma_{i}$.

cells as shown in Fig. 1 with period $L_{y}$. Each cell consists of a volume $\Omega$, the hashed region shown in Fig. 1 containing liquid crystal and a metallic inclusion of volume $\Omega_{n p}$ with boundary $\Gamma_{i}$, where the subscript $i$ labels the inclusions. We label the external boundary of a cell $\mathcal{B}$. The macroscopic domain $\mathcal{D}$ consists of an array of unit cells, with macroscopic boundary $\partial \mathcal{D}$. The system has a separation of scales which we quantify by the smallness parameter $\eta=\frac{L_{y}}{L_{x}}$, where $L_{x}$ is the size of the macroscopic system. The homogenization process involves studying the limiting behavior of a system as $\eta$ tends to zero while the total size of the system remains constant. We consider a system of constant size $L_{x}$ as the size of the periodic microstructure $L_{y}$ tends to zero and the number of inclusions diverges. The inclusions are assumed to remain in fixed positions and orientations, but we pose no restrictions on their geometry. The total free energy of the nematic is

$$
\tilde{F}=\int_{\mathcal{D}} \tilde{\mathcal{F}}_{B}+\tilde{\mathcal{F}}_{t}+\tilde{\mathcal{F}}_{e} d V+\int_{\cup_{i} \Gamma_{i}} \tilde{\mathcal{F}}_{s} d S .
$$

The bulk elastic $\tilde{\mathcal{F}}_{B}$, thermotropic $\tilde{\mathcal{F}}_{t}$, electrostatic $\tilde{\mathcal{F}}_{e}$, and surface $\tilde{\mathcal{F}}_{S}$ free energy density of the nematic are given by

$$
\begin{aligned}
\tilde{\mathcal{F}}_{B}= & \frac{L_{B}}{2}|\nabla \tilde{\mathcal{Q}}|^{2} \\
\tilde{\mathcal{F}}_{t}= & \frac{1}{2} A\left(T-T^{*}\right) \operatorname{Tr}\left(\tilde{\mathcal{Q}}^{2}\right)-\sqrt{6} B \operatorname{Tr}\left(\tilde{\mathcal{Q}}^{3}\right) \\
& +\frac{1}{2} C\left[\operatorname{Tr}\left(\tilde{\mathcal{Q}}^{2}\right)\right]^{2} \\
\tilde{\mathcal{F}}_{e}= & -\frac{1}{3} \epsilon_{0} \Delta \epsilon \operatorname{Tr}(\tilde{\mathcal{Q}} \tilde{\mathcal{E}}) \\
\tilde{\mathcal{F}}_{S}= & \frac{\tilde{\mu}}{2}\left(\tilde{\mathcal{Q}}-\tilde{\mathcal{Q}}_{S}\right)^{2} .
\end{aligned}
$$

Here $A, B$, and $C$ are the Landau coefficients which are assumed to be temperature independent. All temperature effects are confined to the $T-T^{*}$ term, where $T$ is the temperature and $T^{*}$ is the pseudocritical temperature at which the isotropic phase becomes unstable. $L_{B}=\frac{K}{3 \tilde{S}^{2}}$ where $K$ is the elastic constant of the liquid crystal, $\Delta \epsilon$ is the dielectric anisotropy, and $\epsilon_{0}$ is the vacuum permittivity. $\tilde{\mu}$ is the surface-anchoring 
energy density and $\tilde{\mathcal{Q}}_{S}$ is the preferred alignment tensor which parametrizes the surface free energy. The electrostatic tensor $\tilde{\mathcal{E}}$ is related to the electric potential by

$$
\tilde{\mathcal{E}}=\sqrt{\frac{3}{2}}\left[\nabla \tilde{\phi} \otimes \nabla \tilde{\phi}-\frac{\mathcal{I}}{3}\right],
$$

where $\tilde{\phi}$ is the electric potential. We follow the approach outlined in Ref. [29] and express the scaled $\mathcal{Q}$ tensor, preferred alignment tensor, and electrostatic tensor on the basis of traceless symmetric tensors. The components on this basis are

$$
\begin{aligned}
\tilde{a}_{m} & =\operatorname{Tr}\left[\mathcal{T}^{(m)} \tilde{\mathcal{Q}}\right], \\
\tilde{e}_{m} & =\operatorname{Tr}\left[\mathcal{T}^{(m)} \tilde{\mathcal{E}}\right], \\
\tilde{a}_{S m} & =\operatorname{Tr}\left[\mathcal{T}^{(m)} \tilde{\mathcal{Q}}_{S}\right],
\end{aligned}
$$

which form the $m$ th components of the vectors $\tilde{\boldsymbol{a}}, \tilde{\boldsymbol{e}}$ and $\tilde{\boldsymbol{a}}_{S}$, where $\mathcal{T}^{(m)}, m=1, \ldots, 5$ are a basis of traceless symmetric tensors defined by Eqs. (A1) in the Appendix.

We are interested in the case where neither the surface energy nor the bulk energy dominates the behavior of the system. We expect that in this regime there will be a richer interplay between the two constituents, particles and liquid crystal, that will show the potential of homogenization theory to obtain realistic macroscopic equations. To this end we require that the ratio of bulk to surface energy remains constant as $\eta$ tends to zero. This imposes constraints on the strength of the surface anchoring in the system because the total surface area of the inclusions tends to infinity as $\eta$ tends to zero. To determine the scaling of the anchoring energy we estimate the total bulk elastic and surface energy in the system. For the system pictured in Fig. 1 the dimensional bulk elastic energy $\tilde{F}_{B}$ and surface energy $\tilde{F}_{S}$ are given by

$$
\begin{aligned}
& \tilde{F}_{B}=\int_{D} \frac{L_{B}}{2}|\nabla \tilde{\mathcal{Q}}|^{2} d V \sim V_{l c} \frac{L_{B}}{2}, \\
& \tilde{F}_{S}=\sum_{i} \int_{\Gamma_{i}} \frac{\tilde{\mu}}{2}\left(\tilde{\mathcal{Q}}-\tilde{\mathcal{Q}}_{S}\right)^{2} d S \sim A_{\Gamma} \frac{\tilde{\mu}}{2},
\end{aligned}
$$

with $V_{l c}$ being the total volume of liquid crystal and $A_{\Gamma}$ being the total contact area with the inclusions. For spherical inclusions of radius $r=\eta r_{0}$, the total surface area of the inclusions is $A_{\Gamma}=4 \pi r_{0}^{2} \eta^{2} N$ where $N$ is the total number of inclusions. The total number of inclusions in the system is $N=\frac{1}{\eta^{3}}$, hence the surface energy $\tilde{F}_{S}$ scales as $\tilde{\mu} \eta^{-1}$. The total bulk elastic energy is independent of $\eta$, hence, for a system with a constant ratio of surface to bulk free energy, the anchoring energy density $\tilde{\mu}$ must scale as $\eta$. This constraint is compatible with the requirement that the anchoring is weak enough not to induce defects in the particle neighborhood. As shown by Ref. [30] no defects form if $\tilde{\mu} r / L_{B} \ll 4$. As both $\tilde{\mu}$ and $r$ scale with $\eta$ this relation is always satisfied in the homogenization limit, $\eta \rightarrow 0$.

Finally, we assume that the inclusions can be treated as ideal conductors and solve Maxwell's equations subject to a constant floating potential across each individual inclusion. This approximation is justified for dopant particles considered here under ac conditions, because the penetration depth of the electric field is far smaller than the particles.
Details of the nondimensionalization used are given in the Appendix. The governing equations can be derived from a dissipation principle [31]. When fluid flow is neglected the dissipation principle takes the form

$$
\frac{\partial \mathcal{R}}{\partial a_{i, \tilde{t}}}=\frac{\partial}{\partial \tilde{x}_{j}} \frac{\tilde{F}_{V}}{\partial a_{i, j}}-\frac{\partial \tilde{F}_{V}}{\partial a_{i}},
$$

with the dissipation function given by

$$
\mathcal{R}=\frac{1}{2} \zeta\left(\frac{\partial \boldsymbol{a}}{\partial \tilde{t}}\right)^{2}
$$

Here $\zeta$ is related to the Leslie rotational viscosity $\gamma_{1}$ by $\zeta=$ $\frac{\gamma_{1}}{3 \tilde{S}^{2}}, \tilde{t}$ is time, and $\tilde{F}_{V}$ is the total volume free energy

$$
\tilde{F}_{V}=\int_{\mathcal{D}}\left(\tilde{\mathcal{F}}_{e}+\tilde{\mathcal{F}}_{t}+\tilde{\mathcal{F}}_{B}\right) d V
$$

In the limit of static configurations the dissipative principle produces the Euler-Lagrange equations minimizing the free energy of the nematic. The governing equations are obtained from Eq. (7), and the boundary conditions on the inclusions are time independent and are found from minimizing the free energy over $\cup_{i} \Gamma_{i}$. In nondimensional form these are

$$
\begin{aligned}
\tilde{\xi}_{0}^{2} \nabla^{2} \boldsymbol{a}+\tilde{\chi}_{a} \boldsymbol{e}+M(\boldsymbol{a}) \boldsymbol{a} & =\frac{\partial \boldsymbol{a}}{\partial t}, \quad \boldsymbol{x} \in \mathcal{D}, \\
\hat{\boldsymbol{n}} \cdot \nabla \boldsymbol{a} & =W\left(\boldsymbol{a}_{S}-\boldsymbol{a}\right), \quad \boldsymbol{x} \in \Gamma, \\
\boldsymbol{a} & =\boldsymbol{b}(\boldsymbol{x}), \quad \boldsymbol{x} \in \partial \mathcal{D},
\end{aligned}
$$

where $\boldsymbol{b}(\boldsymbol{x})$ is the prescribed value of $\boldsymbol{a}(\boldsymbol{x})$ on the macroscopic boundary $\partial \mathcal{D}$, and $\hat{\boldsymbol{n}}$ is the unit normal on $\Gamma_{i}$. The nondimensional elastic constant is given by $\tilde{\xi}_{0}^{2}=\frac{9 C L_{B}}{2 B^{2} L_{x}^{2}}, \tilde{\chi}_{a}=\frac{9 \epsilon_{0} \Delta \epsilon C^{2} \Psi_{0}}{2 L_{x}^{2} B^{3}}$ is the dimensionless dielectric anisotropy, $W=\frac{\tilde{\mu} L_{x}}{2 L_{B}}$ is the dimensionless anchoring strength, and $\Psi_{0}$ is a characteristic potential. The nondimensional time $t$ and spatial position $\boldsymbol{x}$ are $t=\frac{2 B^{2}}{9 C \zeta} \tilde{t}$ and $\tilde{\boldsymbol{x}}=L_{x} \boldsymbol{x}$, with $\tilde{\boldsymbol{x}}$ being the dimensional spatial position. The nonlinear thermotropic contribution to the EulerLagrange equations are confined to the thermotropic matrix $M(\boldsymbol{a})$ given by Eq. (A5). We assume homeotropic anchoring on the inclusions throughout this paper. The governing equation in the bulk [Eq. (10a)] reduces to the Euler-Lagrange equation when stationary configurations are considered. The electric field is governed by Maxwell's equation $\boldsymbol{\nabla} \cdot \boldsymbol{D}=0$. We model the gold inclusions as macroscopic ideal conductors, ignoring finite-size effects. The electric potential is hence subject to a floating potential on the metallic inclusions. Expressed in terms of the nondimensional electrostatic potential $\phi$, this reads

$$
\begin{aligned}
\nabla \cdot[(\mathcal{I}+\alpha \mathcal{Q}) \nabla \phi] & =0, \quad \boldsymbol{x} \in \mathcal{D}, \\
\phi & =V_{0}(\boldsymbol{x}), \quad \boldsymbol{x} \in \partial \mathcal{D}, \\
\phi & =\mathrm{C}_{i}, \quad \boldsymbol{x} \in \Gamma_{i}, \\
\int_{\Gamma_{i}} \hat{\boldsymbol{n}} \cdot[(\mathcal{I}+\alpha \mathcal{Q}) \nabla \phi] d S & =0, \quad \boldsymbol{x} \in \Gamma_{i},
\end{aligned}
$$

where

$$
\alpha=\sqrt{\frac{2}{3}} \frac{\Delta \epsilon}{\epsilon_{u}} \frac{2 B}{3 C}
$$


TABLE I. Parameters used in numerical simulations; values represent a typical liquid crystal.

\begin{tabular}{lc}
\hline$K=15 \mathrm{pN}$ & $S=\frac{3+\sqrt{9-8 T_{0}}}{4}$ \\
$A=0.13 \times 10^{6} \mathrm{~J} \mathrm{~K}^{-1} \mathrm{~m}^{-3}$ & $\epsilon_{\perp}=4.1$ \\
$B=1.6 \times 10^{6} \mathrm{~J} \mathrm{~K}^{-1} \mathrm{~m}^{-3}$ & $\epsilon_{\|}=9.1$ \\
$C=3.9 \times 10^{6} \mathrm{~J} \mathrm{~K}^{-1} \mathrm{~m}^{-3}$ & $L_{x}=32 \times 10^{-6} \mathrm{~m}$ \\
$T_{0}=-10$ & $\gamma_{1}=0.081 \mathrm{~Pa} \mathrm{~s}$ \\
$\tilde{\mu}=3.23 \times 10^{-8} \mathrm{~W} \mathrm{~J} / \mathrm{m}^{2}$ & $\tilde{\xi}_{0}^{2}=4.631 \times 10^{-8}$ \\
$\tilde{\chi}_{a}=7.221 \times 10^{-7}$ & $\alpha=0.1936$ \\
\hline \hline
\end{tabular}

with $\epsilon_{u}$ being the isotropic dielectric coefficient. $C_{i}$ is the constant potential attained on the surface $\Gamma_{i}$ of the $i$ th inclusion. The value of this constant is determined by the macroscopic boundary conditions $(11 \mathrm{~b}) . V_{0}(\boldsymbol{x})$ is the prescribed value of the potential on the macroscopic boundary $\partial \mathcal{D}$.

\section{B. A priori estimates}

The nondimensional elastic constant $\tilde{\xi}_{0}^{2}$ and dielectric anisotropy $\tilde{\chi}_{a}$ are very small. For a typical liquid crystal mixture with parameters given by Table I $\tilde{\xi}_{0}^{2}=4.631 \times 10^{-8}$ and $\tilde{\chi}_{a}=7.221 \times 10^{-7}$. Conversely, the thermotropic term $M(\boldsymbol{a}) \boldsymbol{a}$ is $O(1)$, indicating that the liquid crystal dynamics, Eq. (10a), has at least two different timescales [32]. Evolution on the fast timescale is determined by the thermotropic term $M(\boldsymbol{a}) \boldsymbol{a}$ which drives the $\mathcal{Q}$ tensor into the uniaxial state [32]. We are interested in the reorientation of the liquid crystal due to the elastic and electrostatic energies. The re-orientational dynamics occurs on a far slower timescale because it is driven by the weaker elastic and electrostatic terms. In a typical setup, $L_{x} \geqslant 10 \mu \mathrm{m}, L_{y} \leqslant 100 \mathrm{~nm}$ and, hence, $\eta \leqslant 0.01$. We therefore scale the liquid crystal parameters as $\tilde{\xi}_{0}^{2}=\eta^{2} \xi_{0}^{2}$ and $\tilde{\chi}_{a}=\eta^{2} \chi_{a}$. As the slow time dynamics is driven by these two $O\left(\eta^{2}\right)$ quantities, we rescale time with $\eta^{2}$,

$$
\frac{\partial}{\partial t}=\eta^{2} \frac{\partial}{\partial t_{2}}
$$

and assume that all faster processes have reached equilibrium. The timescale $t_{2}$, given by Eq. (12), is the slow timescale on which the nematic reorientates.

The last scaling relation that we need to introduce concerns the surface-anchoring energy. As discussed earlier, in this paper we consider the case where the ratio of surface to bulk energy remains constant as $\eta \rightarrow 0$. We have shown, by using Eqs. (6), that this implies that the surface-anchoring energy $W$ must scale linearly with $\eta$. We therefore define a scaled anchoring energy $W_{1}$ as $W=\eta W_{1}$.

Before we proceed to apply homogenization theory to our system we identify the physically relevant range of anchoring energies that our model applies to. We assume inclusions are fixed and do not nucleate defects. As noted previously, the weak-anchoring condition [30] always holds in the homogenization limit. Assuming fixed inclusions is likely to be valid provided inclusions do not aggregate on the experimental timescale. In a real system $\eta \neq 0$, hence, we now estimate values of $W_{1}$ that do not induce defects.

Using the parameters in Table I we find that the anchoring energy density scales as $\tilde{\mu}=3.28 \times 10^{-8} \eta W_{1} \mathrm{~J} / \mathrm{m}^{2}$. For a typical liquid crystal cell used in our numerical simulations $L_{x}=32 \mu \mathrm{m}, L_{y}=1 \mu \mathrm{m}$, and a particle of radius $50 \mathrm{~nm}$, weak anchoring corresponds to $W_{1} \ll 40000$.

Ruhwandl et al. [7] identify the aggregation time using a director based model by considering the elastic interaction between separated colloidal particles. The aggregation time is given by [7]

$$
\tau_{a g}=\frac{0.5 L_{B} \nu}{\tilde{\mu}^{2}}\left(\frac{1}{c^{7 / 3}}-\frac{1}{c_{m}^{7 / 3}}\right),
$$

where $v$ is a characteristic viscosity taken to be $v=0.1 \mathrm{~Pa} \mathrm{~s}$ and $c_{m}=0.74$ is the maximum colloidal packing fraction. Using Table I and the above parameters, the volume fraction of particles is $c=5 \times 10^{-4}$ giving an aggregation of $\tau_{a g} \approx 25$ weeks for $W_{1}=100$. We expect the theory developed here to apply to low volume concentrations, i.e., $c \leqslant 0.01$. However, for the purpose of numerical verification it is easier to simulate systems containing larger inclusions, and hence larger volume fractions.

\section{Homogenization}

To homogenize Eqs. 10(c) and 11(d) we use a perturbation expansion in the small parameter $\eta$. We define a microscopic scale $\boldsymbol{y}$ by the relation $\boldsymbol{y}=\frac{\boldsymbol{x}}{\eta}$ and assume that all fields are, $a$ priori, functions of $\boldsymbol{x}$ and $\boldsymbol{y}$. We use the chain rule and expand $\phi, \boldsymbol{a}, \mathcal{Q}, \boldsymbol{e}$, the floating potentials $C_{i}$, and the spatial derivatives as a series in $\eta$ :

$$
\begin{aligned}
\nabla & =\frac{1}{\eta} \nabla_{\boldsymbol{y}}+\nabla_{\boldsymbol{x}}, \\
\boldsymbol{a} & =\boldsymbol{a}_{0}+\eta \boldsymbol{a}_{1}+\eta^{2} \boldsymbol{a}_{2}+O\left(\eta^{3}\right), \\
\phi & =\phi_{0}+\eta \phi_{1}+\eta^{2} \phi_{2}+O\left(\eta^{3}\right), \\
\mathcal{Q} & =\mathcal{Q}_{0}+\eta \mathcal{Q}_{1}+\eta^{2} \mathcal{Q}_{2}+O\left(\eta^{3}\right), \\
\boldsymbol{e} & =\frac{\boldsymbol{e}_{0}}{\eta^{2}}+\frac{\boldsymbol{e}_{1}}{\eta}+\boldsymbol{e}_{2}+O(\eta), \\
C_{i} & =C_{0 i}+\eta C_{1 i}+\eta^{2} C_{2 i}+O\left(\eta^{3}\right),
\end{aligned}
$$

where $\boldsymbol{e}_{0}, \boldsymbol{e}_{1}$, and $\boldsymbol{e}_{2}$ have components given by Eq. (A8c).

Here we provide an overview of the homogenization process, with full details given in the Appendix. First we substitute the ansatz (14) into Eqs. 10(c) and 11(d). This produces a set of equations at different order in $\eta$ which we solve on the microscale in ascending powers of $\eta$ up to and including terms of $O\left(\eta^{2}\right)$. To first approximation, i.e., collecting terms of $O\left(\eta^{0}\right)$, we see that variations in the electric potential or alignment of the liquid crystal on the scale $L_{x}$ are neglected and the macroscopic boundary conditions do not come into the problem. Because the alignment on the inclusions is weak there is nothing to fix the liquid crystal orientation at this order. The result is that $\boldsymbol{a}_{0}$ is independent of the microscale coordinate $\boldsymbol{y}$ with the constraint that $\boldsymbol{a}_{0}$ is uniaxial with scalar order parameter determined by the temperature of the system. Similarly we find that $\phi_{0}$ is only fixed by the boundary condition on the inclusion, Eq. (11c). Hence, $\phi_{0}$ must be independent of position and must define the value of the potential on the particle surface.

In order to uniquely determine the macroscale dependence of the variables $\boldsymbol{a}_{0}$ and $\phi_{0}$, we collect terms at $O(\eta)$. However, 
as is common to homogenization problems, rather than finding an equation for $\boldsymbol{a}_{0}(\boldsymbol{x})$ and $\phi_{0}(\boldsymbol{x})$, we find a set of equations which relate the derivatives of these quantities on the scale $L_{x}$ to the first-order corrections $\boldsymbol{a}_{1}$ and $\phi_{1}$. These equations are commonly referred to as cell problems. Unlike the equations at $O\left(\eta^{0}\right)$, we find that these cell problems have a solution which depends on both $\boldsymbol{x}$ and $\boldsymbol{y}$. Using an appropriate change of variable, we are able to solve these cell problems to find the relationship between $\boldsymbol{a}_{1}$ and $\nabla_{x} \boldsymbol{a}_{0}$. Similarly, we obtain a relationship between $\phi_{1}$ and $\nabla_{x} \phi_{0}$. Note, however, that we do not need to explicitly obtain $\boldsymbol{a}_{1}$ and $\phi_{1}$.

Finally, we expand to $O\left(\eta^{2}\right)$ and obtain an equation for $\boldsymbol{a}_{2}$ and $\phi_{2}$. Rather than solve these equations directly we impose a solvability condition to eliminate $\boldsymbol{a}_{2}$ and $\phi_{2}$. The result is an averaged set of equations for $\boldsymbol{a}_{0}$ and $\phi_{0}$ which is independent of the microscale geometry. These equations capture the microscopic geometric features through a set of effective parameters which are obtained from the cell problems found at $O\left(\eta^{1}\right)$. In addition, the effect of the anchoring to the inclusions is captured through an averaged forcing term. Combined with the $O\left(\eta^{0}\right)$ constraints and macroscopic boundary conditions, these equations form a complete description of the liquid crystal alignment. The homogenized equations are

$$
\begin{aligned}
\boldsymbol{V}^{(k)} \cdot \frac{\partial \boldsymbol{a}_{0}}{\partial t_{2}}= & \boldsymbol{V}^{(k)} \cdot\left\{\xi_{0}^{2} \nabla_{\boldsymbol{x}} \cdot\left(D \nabla_{\boldsymbol{x}} \boldsymbol{a}_{0}\right)\right. \\
& \left.+\chi_{a}\left[\boldsymbol{e}_{M}\left(1+2 \frac{\left|\Omega_{n p}\right|}{|\Omega|}\right)+\boldsymbol{p}\right]+W_{1} \xi_{0}^{2} \boldsymbol{q}\right\},
\end{aligned}
$$

$$
\nabla_{x} \cdot\left[\left(|\Omega|+\left|\Omega_{n p}\right|\right)\left(\mathcal{I}+\alpha \mathcal{Q}_{0}\right) \nabla_{x} \phi_{0}\right]=0,
$$

for $k=1,2,3$ where $\boldsymbol{V}^{(k)}$ are given by Eq. (A19) and span the tangent space of the manifold of uniaxial $\mathcal{Q}$ tensors [32]. These equations are solved subject to the constraint that the scalar order parameter is a constant determined by the temperature of the nematic and the biaxiality is zero.

The effective fields and material parameters that appear in Eqs. (15) are given by

$$
\begin{aligned}
D_{i j}= & \frac{1}{|\Omega|} \int_{\Omega}\left(\delta_{i j}+\frac{\partial \chi_{j}}{\partial y_{i}}\right) d^{3} y, \\
\boldsymbol{q} & =\frac{1}{|\Omega|} \int_{\Gamma} \boldsymbol{a}_{S} d S, \\
e_{M i}= & \operatorname{Tr}\left[\nabla_{\boldsymbol{x}} \phi_{0} \otimes \nabla_{\boldsymbol{x}} \phi_{0} \mathcal{T}^{(i)}\right], \\
p_{i}= & \frac{1}{|\Omega|} \int_{\Omega} \operatorname{Tr}\left[\left(\sum_{k=1}^{3} \nabla_{\boldsymbol{y}} R_{k} \frac{\partial \phi_{0}}{\partial x_{k}}\right)\right. \\
& \left.\otimes\left(\sum_{k=1}^{3} \nabla_{\boldsymbol{y}} R_{k} \frac{\partial \phi_{0}}{\partial x_{k}}\right) \mathcal{T}^{(i)}\right] d V .
\end{aligned}
$$

Here, $|\Omega|$ is the volume of liquid crystal in a unit cell, $\left|\Omega_{n p}\right|$ is the volume occupied by an inclusion, and $\delta_{i j}$ is the Kronecker delta. The fields $\chi_{k}(\boldsymbol{y})$ and $R_{k}(\boldsymbol{y}), k=1,2,3$ that appear in Eqs. (16) and (19) are the solutions of the two sets of cell problems.
The first set of cell problems determine $\chi_{k}, k=1,2,3$ and relates the change in elastic response of the system to the microscopic geometry:

$$
\begin{aligned}
\xi_{0}^{2} \nabla_{y}^{2} \chi_{k} & =0, \quad \boldsymbol{y} \in \Omega, \\
\hat{\boldsymbol{n}} \cdot \nabla_{\boldsymbol{y}} \chi_{k} & =-\hat{\boldsymbol{n}} \cdot \hat{\boldsymbol{e}}_{k}, \quad \boldsymbol{y} \in \Gamma_{i},
\end{aligned}
$$

where $\hat{\boldsymbol{e}}_{k}$ for $k=1,2,3$ are the Cartesian basis vectors. Although Eqs. (20) define $\chi_{k}$ up to a constant, only the derivative of $\chi_{k}$ enters the homogenized equations. In the appendix, where we give more details of the derivation of the cell problem, we also show a plot of $\chi_{3}$ for an arbitrary value of the constant (Fig. 6).

The second set of cell problems determine the altered electric field felt by the nematic due to the presence of the metallic inclusions:

$$
\begin{aligned}
{\left[\delta_{i j}+\alpha \mathcal{Q}_{0 i j}\right] \frac{\partial^{2} R_{k}}{\partial y_{i} \partial y_{j}} } & =0, \quad \boldsymbol{y} \in \Omega, \\
R_{k} & =-\left(y_{k}-y_{0 k}\right), \quad \boldsymbol{y} \in \Gamma_{i} \\
\int_{\Gamma_{i}}\left(\mathcal{I}+\alpha \mathcal{Q}_{0}\right) \nabla_{\boldsymbol{y}} R_{k}(\boldsymbol{y}) \cdot d \boldsymbol{S} & =0, \quad \boldsymbol{y} \in \Gamma_{i} .
\end{aligned}
$$

When supplemented with macroscopic boundary conditions on $\partial \mathcal{D}$, Eqs. (15)-(21) form a complete macroscopic description of the liquid crystal alignment.

In the next section we show that Eqs. (15)-(21) are both accurate and numerically efficient. Before doing this, however, we want to highlight that they also give a clear, quantitative, and deep physical insight into the behavior of the doped liquid crystal. The material parameters and effective fields defined by Eqs. (16)-(19) have the following physical interpretations: Under the single elastic constant approximation the elasticity of the undoped nematic is isotropic. This is not true in general when inclusions are present. The effective elasticity tensor $D_{i j}$ encodes the anisotropic excluded volume effect into the homogenized equations. We assume homeotropic anchoring on the surface of the inclusions which remain in fixed positions and orientations. This results in a forcing term $\boldsymbol{q}$ that favors a nematic aligned with the average preferred orientation on the inclusions. We modeled the inclusions as ideal metallic particles, assuming the potential is a constant value across each inclusion. Hence the macroscopic electric field $\boldsymbol{e}_{M}$ is amplified by the factor $\left(1+2 \frac{\left|\Omega_{n p}\right|}{|\Omega|}\right)$. This factor quantifies the increase in voltage drop due to the constant potential on the metallic inclusions. Finally, the polarization field $\boldsymbol{p}$ results from the fringe electric fields around an inclusion: the electric field must be orthogonal to the surface of the inclusions. Hence, even a uniform external electric field will induce components along all the coordinate axes close to the inclusions.

\section{ANALYSIS OF MODEL}

\section{A. Validation}

In order to validate Eqs. (15)-(21) we compare their predictions with numerical simulations of the full system for two cases of inclusions in a twisted cell. In a twisted cell, the director is parametrized by $\hat{d}=$ 

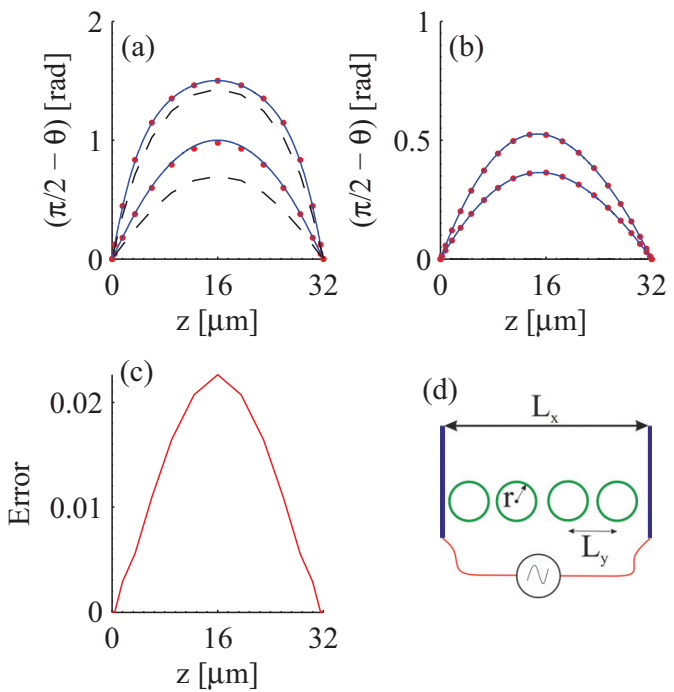

(d)

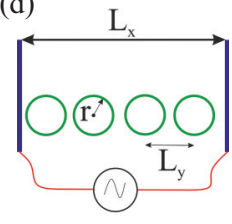

FIG. 2. (Color online) Comparison of numerical simulation of the full and homogenized equations for spherical and ellipsoidal particles inside a twisted geometry, zero pretilt at boundaries, and homeotropic anchoring on dopants. (a) Spherical particles of radius $r=0.3 \mu \mathrm{m}$ at applied voltages of 1.5 and $3 \mathrm{~V}$ (lower and upper curves, respectively). (b) Ellipsoidal dopants $V=0$ with anchoring energy $W_{1}=50$ (bottom) and $W_{1}=100$ (top) orientation given by $\left(\theta_{p}, \phi_{p}\right)=\left(45^{\circ}, 30^{\circ}\right)$. The semimajor axis of the ellipsoids is $0.3 \mu \mathrm{m}$ and the two minor axes are $0.1 \mu \mathrm{m}$. [(a), (b)] Red points are from homogenization, broken black line is undoped, and blue line is from COMSOL numerical simulations. (c) Absolute error $\mathbb{E}=\left|\theta_{H}-\theta_{N}\right|$ where $\theta_{H}$ is the tilt angle from the homogenized equations and $\theta_{N}$ is extracted from numerical simulations of the full system. (d) Schematic diagram of the system studied: planar cell of size $L_{x}$ with spherical or ellipsoidal dopants with spacing $L_{y}$.

$\left(\sin \left(\theta_{H}\right) \cos \left(\phi_{H}\right), \sin \left(\theta_{H}\right) \sin \left(\phi_{H}\right), \cos \left(\theta_{H}\right)\right)$ (see Ref. [33], page 101) with the $x_{3}$ axis measuring distance into the cell. The tilt angle $\theta_{H}$ is measured down from the $x_{3}$ axis and the twist angle $\phi_{H}$ is measured counterclockwise from the $x_{1}$ axis. The first case consists of spherical particles with an applied electric field and the second case consists of ellipsoidal particles in the absence of an applied field; see Fig. 2. The cells are of thickness $L_{x}=32 \mu \mathrm{m}$, the particles are assumed to be evenly distributed with separation $L_{y}=1 \mu \mathrm{m}$, and the material parameters used are given in Table I. In the first case, the spherical particles are of radius $0.3 \mu \mathrm{m}$; in the second case the semimajor axis of the ellipsoids is $0.3 \mu \mathrm{m}$ and the two minor axes are $0.1 \mu \mathrm{m}$, the ellipsoids orientation is given by $\left(\theta_{p}, \phi_{p}\right)=\left(45^{\circ}, 30^{\circ}\right)$. With $\theta_{p}$ measured down from the $x_{3}$ axis and the twist angle $\phi_{p}$ measured counterclockwise from the $x_{1}$ axis.

We apply a potential difference across the liquid crystal (LC) cell and compare the director profile obtained by solving the full system of equations $11(\mathrm{~d})$ and $10(\mathrm{c})$ using COMSOL multiphysics, a finite element package, to that obtained from the approximate system, Eq. (15). The full $\mathcal{Q}$-tensor equations were implemented as a general form partial differential equation (PDE) in COMSOL. The geometry consists of an array of unit cells along the $x_{3}$ axis, each containing a single inclusion. We imposed periodic boundary conditions on the exterior sides parallel to the $x_{3}$ axis and strong-anchoring conditions with zero pretilt and pretwist on the sides parallel to the $x_{1}-x_{2}$ plane. A free tetrahedral mesh of custom mesh size was used in the bulk of the domain and a free triangular mesh was applied at the boundaries. We used the time-dependent solver with relative and absolute tolerances of $10^{-4}$, the default MUMPS linear system solver and the solution to the homogenized equations as initial conditions. To solve the homogenized equations we used a pseudospectral code as described in Ref. [32] and we solved the cell problems (20) and (21) using COMSOL. To compute Eq. (19) the cell problem (21) must be solved as $\mathcal{Q}_{0}$ varies throughout the cell. We did this by solving Eq. (21) for a range of values of $\mathcal{Q}_{0}$ and interpolating. We also computed the response of an undoped cell for comparison by using a spectral method. Figure 2 shows a comparison between the homogenization predictions, the full finite element simulation, and an undoped solution corresponding to the case where no particles are present. We see that there is very good agreement between the homogenized equations and numerical simulations for both spherical and ellipsoidal particles.

\section{B. Doped liquid crystals in a splay geometry}

Having validated the homogenized equations, we now use them to study the effect of dopants on two characteristic features of liquid crystal cells in a splay geometry; namely, the Fredericks transition, and the switch-on and switch-off times. In the splay geometry the director is confined to the $x_{1}-x_{3}$ plane and varies only in the $x_{3}$ direction with $\tilde{x}_{3} \in\left[0, L_{x}\right]$. The director is parametrized by the angle $\theta_{H}\left(x_{3}, t\right)$ measured from the $x_{3}$ axis, $\hat{\boldsymbol{d}}=\left(\sin \left(\theta_{H}\right), 0, \cos \left(\theta_{H}\right)\right)$; with this parametrization the constraints on the order parameters are automatically met. The governing equation (15a) reduces to a zero twist constraint and a governing PDE for the director angle $\theta_{H}$. The director angle satisfies

$$
\begin{aligned}
\frac{\partial \theta_{H}}{\partial t}= & \xi_{0}^{2} D_{33} \frac{\partial^{2} \theta_{H}}{\partial x_{3}^{2}}+\frac{\chi_{a}}{2 S_{0}}\left(\frac{\sqrt{3}}{3} F_{2}\left(\theta_{H}\right)-F_{1}\left(\theta_{H}\right)\right) \sin \left(2 \theta_{H}\right)\left(\frac{\partial \phi_{0}}{\partial x_{3}}\right)^{2}+\frac{\chi_{a} \sqrt{3}}{3 S_{0}} \cos \left(2 \theta_{H}\right) F_{4}\left(\theta_{H}\right)\left(\frac{\partial \phi_{0}}{\partial x_{3}}\right)^{2} \\
& +\frac{\xi_{0}^{2} W}{3 S_{0}} \sin \left(2 \theta_{H}\right)\left(\frac{\sqrt{3}}{2} q_{2}-\frac{3}{2} q_{1}\right)+\frac{\sqrt{3} \xi_{0}^{2} W}{3 S_{0}} \cos \left(2 \theta_{H}\right) q_{4}, \\
0= & {\left[\xi_{0}^{2} W q_{3}+\chi_{a} F_{3}\left(\theta_{H}\right)\left(\frac{\partial \phi_{0}}{\partial x_{3}}\right)^{2}\right]\left[\cos ^{2}\left(\theta_{H}\right)-1\right]-\frac{\sin \left(2 \theta_{H}\right)}{2}\left[\xi_{0}^{2} W q_{5}+\chi_{a} F_{5}\left(\theta_{H}\right)\left(\frac{\partial \phi_{0}}{\partial x_{3}}\right)^{2}\right] }
\end{aligned}
$$




$$
\begin{aligned}
0 & =\left\{\chi_{a}\left[\sin \left(\theta_{H}\right) F_{3}\left(\theta_{H}\right)+\cos \left(\theta_{H}\right) F_{5}\left(\theta_{H}\right)\right]\left(\frac{\partial \phi_{0}}{\partial x_{3}}\right)^{2}+\xi_{0}^{2} W\left[q_{3} \sin \left(\theta_{H}\right)+q_{5} \cos \left(\theta_{H}\right)\right]\right\} \cos \left(\theta_{H}\right), \\
\theta_{H}\left(0, t_{2}\right) & =\theta_{H}\left(1, t_{2}\right)=\frac{\pi}{2},
\end{aligned}
$$

where $D_{33}$ is defined in Eq. (16). Both $D_{33}$ and $q_{i}, i=1, \ldots, 5$ may be computed once the cell problem (20) is solved using COMSOL. The functions $F_{i}\left(\theta_{H}\right), i=1, \ldots, 5$ are given by

$$
\begin{aligned}
F_{1}\left(\theta_{H}\right)= & +\frac{1}{|\Omega|} \int_{\Omega}\left[-\frac{1}{2}\left(\frac{\partial R_{3}}{\partial y_{1}}\right)^{2}-\frac{1}{2}\left(\frac{\partial R_{3}}{\partial y_{2}}\right)^{2}\right. \\
& \left.+\left(\frac{\partial R_{3}}{\partial y_{3}}\right)^{2}+2\left(\frac{\partial R_{3}}{\partial y_{3}}\right)\right] d V, \\
F_{2}\left(\theta_{H}\right)= & \frac{1}{|\Omega|} \int_{\Omega} \frac{\sqrt{3}}{2}\left[\left(\frac{\partial R_{3}}{\partial y_{1}}\right)^{2}-\left(\frac{\partial R_{3}}{\partial y_{2}}\right)^{2}\right] d V, \\
F_{3}\left(\theta_{H}\right)= & \frac{1}{|\Omega|} \int_{\Omega} \sqrt{3}\left(\frac{\partial R_{3}}{\partial y_{1}}\right)\left(\frac{\partial R_{3}}{\partial y_{2}}\right) d V, \\
F_{4}\left(\theta_{H}\right)= & \frac{1}{|\Omega|} \int_{\Omega} \sqrt{3}\left(\frac{\partial R_{3}}{\partial y_{1}}\right)\left(\frac{\partial R_{3}}{\partial y_{3}}+1\right) d V, \\
F_{5}\left(\theta_{H}\right)= & \frac{1}{|\Omega|} \int_{\Omega} \sqrt{3}\left(\frac{\partial R_{3}}{\partial y_{2}}\right)\left(\frac{\partial R_{3}}{\partial y_{3}}+1\right) d V .
\end{aligned}
$$

We compute Eqs. (23) over a uniformly spaced grid of $\theta_{H}$ values in the interval $[0, \pi / 2]$ and interpolate. We use a spectral collocation method to solve Eq. (15) [34].

We consider two microscopic geometries: a regular array of spheres of radius $R$ and an array of ellipsoids of semi axes $r_{1}, r_{2}, r_{3}$ of various orientations. Figure 3 shows how the Fredericks transition depends on the particle radius for spherical dopants and on the aspect ratio at constant surface area for ellipsoidal dopants. Figure 4 shows how the source term and elasticity tensor vary with particle size and orientation for the systems in Fig. 3. Spherical inclusions always reduce the Fredericks transition: the excluded volume effect reduces the elasticity and the presence of metallic particles forces the potential to drop across a smaller region of liquid crystal, giving a larger field. Moreover, for spherical dopants there is no source term $\boldsymbol{q}$ present to stabilize the zero-voltage configuration. Ellipsoidal particles break the symmetry of the Fredericks bifurcation unless their major axis is aligned parallel or orthogonal to the initial director configuration. The Fredericks transition may be lowered or raised depending on the competition between the excluded volume effects in $D_{i j}$, the source term $\boldsymbol{q}$, and the enhanced electric fields $\boldsymbol{e}_{M}$ and p. By linearising Eq. (22a) about the zero-voltage solution, $\theta_{H}=\pi / 2$, we can obtain an algebraic expression for the Fredericks transition,

$$
V_{C}=\sqrt{\frac{\xi_{0}^{2}\left(3 D_{33} \pi^{2} S_{0}+\sqrt{3} W q_{2}-3 W q_{1}\right)}{\chi_{a}\left(-F_{2} \sqrt{3}+\sqrt{3} \frac{\partial F_{4}}{\partial \theta_{H}}+3 F_{1}\right)_{\theta_{H}=\pi / 2}}} .
$$

This formula is valid only if neither the electric field nor the inclusion alignment field $\boldsymbol{q}$ induce a twist and $\theta_{H}=\pi / 2$ remains a solution. These constraints follow from the governing equations (22). Substituting $\theta_{H}=\pi / 2$ into Eq. (22a), we must have

$$
q_{4}=F_{4}=0 .
$$

The zero twist condition is given by Eqs. (22b) and (22c), these are satisfied for arbitrary $\theta_{H}$ provided

$$
q_{3}=q_{5}=F_{3}=F_{5}=0 .
$$

For ellipsoidal particles, Eq. (26) restricts the major axis of the ellipsoids to the plane containing the director. If the major axis of the ellipsoids were to leave the plane, the preferred anchoring on the inclusions and fringe fields would force the director out of plane and the chosen parametrization would no longer describe the system. The second constraint, Eq. (25), guarantees that there is a perfect bifurcation and is satisfied by ellipsoids with homeotropic anchoring provided they are aligned with the initial director or perpendicular to it. If Eq. (25) is not satisfied, the Fredericks transition is softened as if there were pretilt at the boundaries.

To obtain expressions for the switch-on and -off times from the uniform state, we linearize Eq. (22a) about $\theta_{H}=\pi / 2$ and consider the evolution of a small perturbation satisfying Eq. (22d). For the case of zero applied voltage we obtain the nondimensional switch-off time, while for an applied voltage
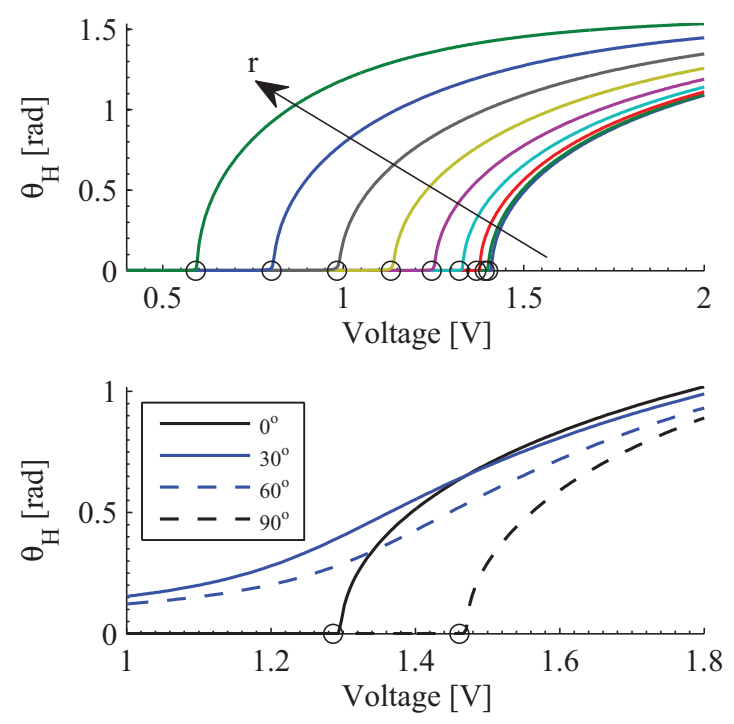

FIG. 3. (Color online) Bifurcation diagrams for spherical and ellipsoidal inclusions. Top spheres of radius from $0.05 \mu \mathrm{m}$ in steps of 0.05 to $0.45 \mu \mathrm{m}$. Bottom: ellipsoids with major axis $r_{1}=0.3 \mu \mathrm{m}$ and minor axis' $r_{2}=r_{3}=0.1 \mu \mathrm{m}$, oriented in the plane of the director at an angle of $0^{\circ}, 30^{\circ}, 60^{\circ}$, and $90^{\circ}$ to the initial director alignment. Circles indicate the position of the Fredericks transition computed using Eq. (24). 


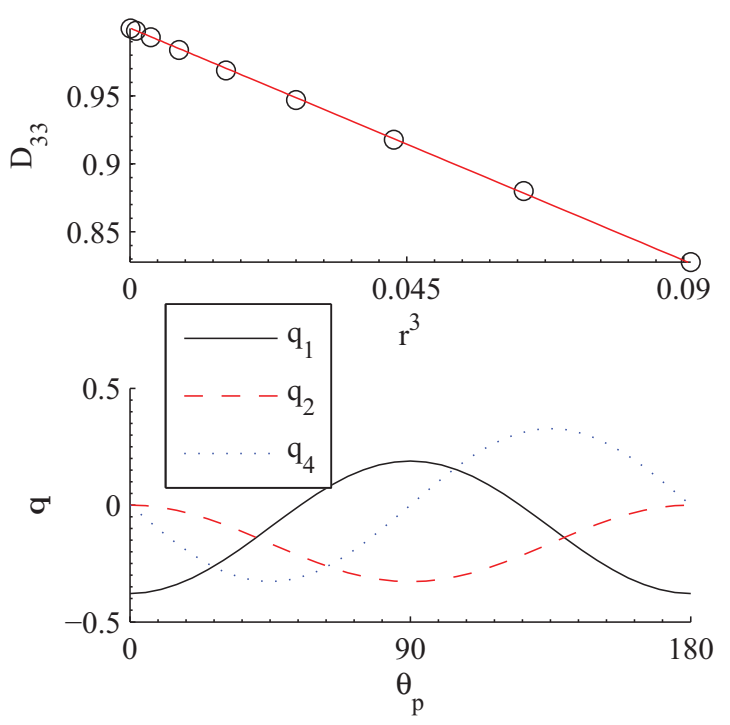

FIG. 4. (Color online) Diagonal component of the elasticity tensor for spherical inclusions and components of the source term $\boldsymbol{q}$ for ellipsoidal particles confined to the $x_{1}-x_{3}$ plane. The angle $\theta_{p}$ is measured from the ellipsoids major axis to the $x_{3}$ axis, $q_{3}=q_{5}=0$ (not shown). The approximate linear relation between $D_{33}$ and $r^{3}$ indicates that the excluded volume effect is dominant.

exceeding the Fredericks threshold we obtain the switch-on time (see Ref. [33], pages 220-222)

$$
\begin{gathered}
\tau_{\mathrm{on}}=\frac{3 S_{0}}{\chi_{a}\left(3 F_{1}-\sqrt{3} F_{2}+\sqrt{3} \frac{\partial F_{4}}{\partial \theta_{H}}\right)_{\theta_{H}=\pi / 2}\left(V^{2}-V_{C}^{2}\right)}, \\
\tau_{\mathrm{off}}=\frac{3 S_{0}}{3 S_{0} \pi^{2} \xi_{0}^{2} D_{33}-\xi_{0}^{2} W\left(3 q_{1}-\sqrt{3} q_{2}\right)} .
\end{gathered}
$$

It should be noted that, even though we have neglected fluid flow in these formulas, the correction to the switch-on times due to induced fluid flow within a planar cell is minimal for most liquid crystals (see Ref. [33], page 228); likewise, the correction to the switch-off time from a slightly distorted state is small. Figure 5 shows how the switching times vary with aspect ratio for a range of ellipsoidal particles with homeotropic anchoring. Particles are either aligned with the initial director or orthogonal to it. In the case of alignment with the initial director, the homeotropic anchoring destabilizes the zero-voltage solution and we see a decrease in the switch-on time and an increase in the switch-off time. This situation is not completely reversed for particles aligned parallel to the initial director. In this case the effective field acts as a restoring force and decreases the switch-off time. However, the enhanced electric field dominates and the switch-on time decreases as well, despite the reduced elastic constant and unfavorable source term. This example illustrates how doping with particles may be used to tailor the properties of the LC to specific applications. The simplicity and computational efficiency of the homogenization method makes optimizing these properties a realistic prospect.

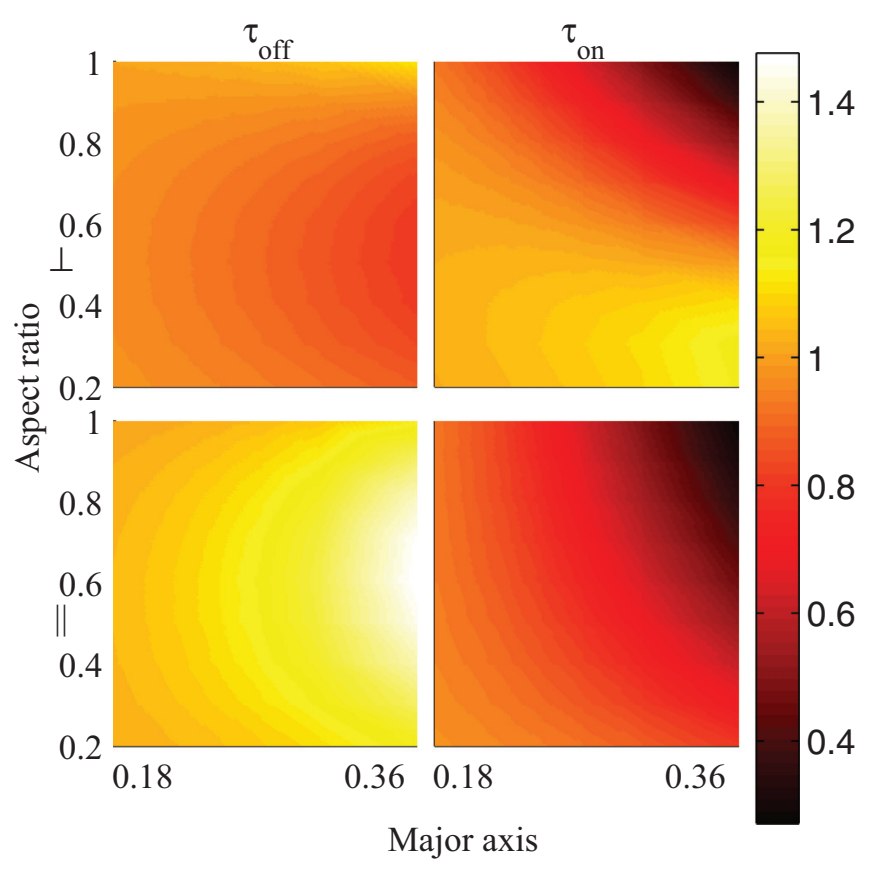

FIG. 5. (Color online) Switch-off (left) and -on (right) time at $V=2 \mathrm{~V}$ for colloidal nematic with ellipsoidal particles as a fraction of undoped on or off times in the splay geometry. Ellipsoids oriented orthogonal (top) or parallel (bottom) to initial director with homeotropic anchoring on inclusions and anchoring strength $W_{1}=10$. Vertical dopants reinforce stability of the $\theta_{H}=0$ state while horizontal dopants reduce it.

\section{CONCLUSIONS}

In this paper we studied the alignment of a nematic liquid crystal which is confined by a set of periodic metal inclusions. By using the method of homogenization, we derived a set of approximate equations which describe the alignment of a liquid crystal subject to an applied external electric field and weak anchoring on the surface of the inclusions. The inclusions we consider are of arbitrary shape but are assumed to vary on a scale much smaller than the macroscopic length scale of interest. Hence, these inclusions may be identified as either a periodic microstructure or a set of dopant particles. The key advantage of this method is that we are able to link the macroscopic properties to the microscale structure through a representative cell problem rather than by explicitly considering the full geometry.

The derivation of these equations is based on several assumptions regarding the physical and geometric properties of the system. Specifically, we consider only metal particles which we consider to be uniformly distributed across the device. The choice of material is not a limitation of the theory developed here and this method could be extended to include dielectric, ferroelectric, or ferromagnetic particles. The result of this would be that the cell problem for the electrostatic field would be different and there would potentially be a coupling between the cell problems for the liquid crystal and electric field. It is also possible to improve upon our existing model for gold. We assumed that the gold inclusions can be modeled as ideal conductors; this could be relaxed to include a finite skin 
depth under dc conditions. Additionally, the optical response of gold nanoparticles of 5 to $100 \mathrm{~nm}$ in diameter can be modeled macroscopically [35]. This is achieved by including scattering contributions to the complex dielectric permittivity. The assumption that the particles are uniformly distributed allows us to simplify the cell problem by using the assumption of periodicity. Similarly assuming that the particles are of fixed position and orientation reduces the interactions that we have to consider.

These approximations are appropriate for the case of a periodically patterned microstructure. However, for the case in which the inclusions take the form of particles, these assumptions may break down and random distributions of particles and particle movement will need to be considered. Random inclusions can be dealt with for low volume fraction materials simply by assuming that any deviation from periodicity will induce an error of $O(\eta)$. However, a more accurate approximation could be obtained by using methods for general random media, such as used, for example, in Ref. [36]. The particle rotation and motion, due to fluid flow or elastic and electrostatic forces, is a more complicated issue. Rigid body rotation of porous structures has been considered in the context of elasticity [37], in which case a nonlinear elasticity theory is derived. The drift of particles could also be included by assuming that the motion is sufficiently slow compared to the reorientation. However, we expect that in this case the correction to our existing results would be small. Dropping the weak-anchoring approximation is another possible extension of this project. Doing so would not be trivial, but may ultimately lead to a detailed comparison to the periodic systems discussed by Muševič et al. [5].

The final homogenized equations (15)-(21) capture the effects of the underlying geometry in terms of an effective elastic tensor and a forcing term which acts to align the liquid crystal to the average preferred direction on the surface of the inclusions. We found excellent agreement between large-scale numerical simulations in which the full geometry is included and the predictions of our model. The homogenized equations require significantly less computation time than the full geometry. The elasticity cell problem for the liquid crystal only needs to be solved once to parametrize the equations and takes less than 10 seconds on a desktop PC for a spherical particle. The cell problem for the electric field (21) must be solved for a range of $\mathcal{Q}_{0}$ to parametrize the equations. However, the solution for each configuration takes under 10 seconds to compute and needs only be done once for any particular dopant. The corresponding simulation of the full geometry takes approximately 15 hours on a single 16 core node of the Iridis 4 supercomputing cluster at the University of Southampton.

By computing the effective material parameters of the nematic as a function of inclusion geometry we can quantify the effect of these inclusions on the Fredericks transition and the switch-on and switch-off times for the liquid crystal. We showed that, by using simple ellipsoidal inclusions with a preferred homeotropic anchoring, the switch-on and switch-off times can be either increased or decreased. The link which this method provides between the macroscopic behavior of the liquid crystal and the underlying geometry will be of fundamental importance in the design of composite liquid crystal materials with optimal material parameters.

\section{ACKNOWLEDGMENTS}

The authors acknowledge the use of the IRIDIS High Performance Computing Facility, and associated support services at the University of Southampton, in the completion of this work. This project was partially supported by the EPSRC Grant No. EP/J006920/1. K.R.D. was funded by BBSRC Grant No. $\mathrm{BB} / \mathrm{J} 000868 / 1$. We are also grateful to Malgosia Kaczmarek and Tim Sluckin for helpful discussions.

\section{APPENDIX: DERIVATION OF EQUATIONS}

\section{Equations for doped liquid crystal}

The basis of traceless symmetric tensors used to define the components of the $\mathcal{Q}$ tensor are

$$
\begin{aligned}
& \mathcal{T}^{(1)}=\frac{1}{\sqrt{6}}\left(-\hat{\boldsymbol{e}}_{1} \otimes \hat{\boldsymbol{e}}_{1}-\hat{\boldsymbol{e}}_{2} \otimes \hat{\boldsymbol{e}}_{2}+2 \hat{\boldsymbol{e}}_{3} \otimes \hat{\boldsymbol{e}}_{3}\right), \\
& \mathcal{T}^{(2)}=\frac{1}{\sqrt{2}}\left(\hat{\boldsymbol{e}}_{1} \otimes \hat{\boldsymbol{e}}_{1}-\hat{\boldsymbol{e}}_{2} \otimes \hat{\boldsymbol{e}}_{2}\right), \\
& \mathcal{T}^{(3)}=\frac{1}{\sqrt{2}}\left(\hat{\boldsymbol{e}}_{1} \otimes \hat{\boldsymbol{e}}_{2}+\hat{\boldsymbol{e}}_{2} \otimes \hat{\boldsymbol{e}}_{1}\right), \\
& \mathcal{T}^{(4)}=\frac{1}{\sqrt{2}}\left(\hat{\boldsymbol{e}}_{1} \otimes \hat{\boldsymbol{e}}_{3}+\hat{\boldsymbol{e}}_{3} \otimes \hat{\boldsymbol{e}}_{1}\right), \\
& \mathcal{T}^{(5)}=\frac{1}{\sqrt{2}}\left(\hat{\boldsymbol{e}}_{2} \otimes \hat{\boldsymbol{e}}_{3}+\hat{\boldsymbol{e}}_{3} \otimes \hat{\boldsymbol{e}}_{2}\right),
\end{aligned}
$$

where $\hat{\boldsymbol{e}}_{i}$ are the unit vectors along the coordinate axis We introduce the scalings $\mathcal{Q}=\frac{3 C}{2 B} \tilde{\mathcal{Q}}, S=\frac{3 C}{2 B} \tilde{S}, \beta=\frac{3 C}{2 B} \tilde{\beta}$, $\tilde{\boldsymbol{\phi}}=\phi \Psi_{0}, \boldsymbol{a}=\frac{3 C}{2 B} \tilde{\boldsymbol{a}}, \boldsymbol{a}_{S}=\frac{3 C}{2 B} \tilde{\boldsymbol{a}}_{S}$, and $\tilde{\boldsymbol{e}}=\boldsymbol{e} \Psi_{0}^{2}$, where $\Psi_{0}$ is a characteristic potential. We scale the spatial coordinates $\tilde{\boldsymbol{x}}=L_{x} \boldsymbol{x}$ where $L_{x}$ is a characteristic length. Expressed in nondimensional form the total volume free energy is

$$
\begin{aligned}
F_{V}= & \int_{\mathcal{D}} \frac{\tilde{\xi}_{0}^{2}}{2}|\nabla \boldsymbol{a}|^{2}+\frac{T_{0}}{2}|\boldsymbol{a}|^{2}+\frac{1}{2}\left|\boldsymbol{a}^{4}\right| \\
& -\sqrt{6} \sum_{p, q, r} \operatorname{Tr}\left(\mathcal{T}^{(p)} \mathcal{T}^{(q)} \mathcal{T}^{(r)}\right) a_{p} a_{q} a_{r}-\tilde{\chi}_{a} \boldsymbol{a} \cdot \boldsymbol{e} d V,
\end{aligned}
$$

with nondimensional anchoring energy

$$
F_{S}=\int_{\cup_{i} \Gamma_{i}} W\left(\boldsymbol{a}-\boldsymbol{a}_{S}\right)^{2} d S .
$$

Here, $\tilde{\xi}_{0}^{2}=\frac{9 C L_{B}}{2 B^{2} L_{x}^{2}}, \tilde{\chi}_{a}=\frac{9 \epsilon_{0} \Delta \epsilon C^{2} \Psi_{0}}{2 L_{x}^{2} B^{3}}, W=\frac{\tilde{\mu} L_{x}}{2 L_{B}}$, and $T_{0}=\frac{T-T^{*}}{T_{c}-T^{*}}$ are the squared of the coherence length, the dielectric coupling constant, the dimensionless anchoring strength, and the reduced temperature, respectively, with $T_{c}$ being the clearing point temperature and the remaining symbols being defined in the main text. The governing equations derived from the dissipation principle (7) and free energy minimization over the boundary of the inclusions are

$$
\begin{aligned}
\tilde{\xi}_{0}^{2} \nabla^{2} \boldsymbol{a}+\tilde{\chi}_{a} \boldsymbol{e}+M(\boldsymbol{a}) \boldsymbol{a} & =\frac{\partial \boldsymbol{a}}{\partial t}, \quad \boldsymbol{x} \in \mathcal{D}, \\
\hat{\boldsymbol{n}} \cdot \nabla \boldsymbol{a} & =W\left(\boldsymbol{a}_{S}-\boldsymbol{a}\right), \quad \boldsymbol{x} \in \Gamma, \\
\boldsymbol{a} & =\boldsymbol{b}(\boldsymbol{x}), \quad \boldsymbol{x} \in \partial \mathcal{D},
\end{aligned}
$$

where $\boldsymbol{b}(\boldsymbol{x})$ is the prescribed value of $\boldsymbol{a}(\boldsymbol{x})$ on the boundary $\partial \mathcal{D}$. Time is scaled according to $\tilde{t}=\frac{9 C}{2 B^{2}} \zeta t$ and $\zeta$ is related to the Leslie rotational viscosity by $\zeta=\frac{\gamma_{1}}{3 \tilde{S}^{2}}$ where $\gamma_{1}$ is the 
rotational viscosity of the liquid crystal. The thermotropic matrix $M$ is given by

$$
M(\boldsymbol{a})=T \mathcal{I}+\left[\begin{array}{ccccc}
3 a_{1} & -3 a_{2} & -3 a_{3} & \frac{3}{2} a_{4} & \frac{3}{2} a_{5} \\
-3 a_{2} & -3 a_{1} & 0 & \frac{3}{2} \sqrt{3} a_{4} & -\frac{3}{2} \sqrt{3} a_{5} \\
-3 a_{3} & 0 & 3 a_{1} & \frac{3}{2} \sqrt{3} a_{5} & \frac{3}{2} \sqrt{3} a_{4} \\
\frac{3}{2} a_{4} & \frac{3}{2} \sqrt{3} a_{4} & \frac{3}{2} \sqrt{3} a_{5} & \frac{3}{2} a_{1}+\frac{3}{2} \sqrt{3} a_{2} & \frac{3}{2} \sqrt{3} a_{3} \\
\frac{3}{2} a_{5} & -\frac{3}{2} \sqrt{3} a_{5} & \frac{3}{2} \sqrt{3} a_{4} & \frac{3}{2} \sqrt{3} a_{3} & \frac{3}{2} a_{1}-\frac{3}{2} \sqrt{3} a_{2}
\end{array}\right],
$$

with

$$
T=-T_{0}-2 \sum_{k=1}^{5} a_{k}^{2} .
$$

The electric field is governed by Maxwell's equation $\boldsymbol{\nabla} \cdot \boldsymbol{D}=$ 0 subject to a floating potential on the metallic inclusions. Expressed in terms of the scaled electrostatic potential $\phi$ this reads

$$
\begin{aligned}
\nabla \cdot[(\mathcal{I}+\alpha \mathcal{Q}) \nabla \phi] & =0, \quad x \in \mathcal{D}, \\
\phi & =V_{0}(x), \quad x \in \partial \mathcal{D}, \\
\phi & =\mathrm{C}_{i}, \quad x \in \Gamma_{i}, \\
\int_{\Gamma_{i}} \hat{\boldsymbol{n}} \cdot[(\mathcal{I}+\alpha \mathcal{Q}) \nabla \phi] d S & =0, \quad x \in \Gamma_{i},
\end{aligned}
$$

where $\alpha=\sqrt{\frac{2}{3}} \frac{\Delta \epsilon}{\epsilon_{u}} \frac{2 B}{3 C}$ with $\epsilon_{u}$ being the isotropic dielectric coefficient. $C_{i}$ is the constant potential attained on the surface $\Gamma_{i}$ of the $i$ th inclusion. It is determined by the macroscopic boundary conditions (A7b). $V_{0}(\boldsymbol{x})$ is the potential on the macroscopic boundary $\partial \mathcal{D}$.

\section{Homogenization}

To homogenize Eqs. (A4c) and (A4d) we use a perturbation expansion in the small parameter $\eta$. We define a microscopic scale $\boldsymbol{y}$ by the relation $\boldsymbol{y}=\frac{\boldsymbol{x}}{\eta}$ and assume that all fields are, a priori, functions of $\boldsymbol{x}$ and $\boldsymbol{y}$. We use the expansions of $\phi$, $\boldsymbol{a}, \mathcal{Q}, \boldsymbol{e}$, the floating potential $C_{i}$, and the spatial derivatives given in Eqs. (14). The terms $\boldsymbol{e}_{0}, \boldsymbol{e}_{1}$, and $\boldsymbol{e}_{2}$ in Eq. (14) have components given by

$$
\begin{aligned}
e_{0 i}= & \operatorname{Tr}\left(\sqrt{\frac{3}{2}}\left[\nabla_{y} \phi_{0} \otimes \nabla_{y} \phi_{0}\right] \mathcal{T}^{(i)}\right), \\
e_{1 i}= & \operatorname{Tr}\left(\sqrt { \frac { 3 } { 2 } } \left[\nabla_{x} \phi_{0} \otimes \nabla_{y} \phi_{0}+\nabla_{y} \phi_{0} \otimes \nabla_{x} \phi_{0}\right.\right. \\
& \left.\left.+\nabla_{y} \phi_{0} \otimes \nabla_{y} \phi_{1}+\nabla_{y} \phi_{1} \otimes \nabla_{y} \phi_{0}\right] \mathcal{T}^{(i)}\right), \\
e_{2 i}= & \operatorname{Tr}\left(\sqrt { \frac { 3 } { 2 } } \left[\nabla_{y} \phi_{0} \otimes\left(\nabla_{y} \phi_{2}+\nabla_{x} \phi_{1}\right)\right.\right. \\
& +\left(\nabla_{x} \phi_{0}+\nabla_{y} \phi_{1}\right) \otimes\left(\nabla_{x} \phi_{0}+\nabla_{y} \phi_{1}\right) \\
& \left.\left.+\left(\nabla_{y} \phi_{2}+\nabla_{x} \phi_{1}\right) \otimes \nabla_{y} \phi_{0}\right] \mathcal{T}^{(i)}\right),
\end{aligned}
$$

for $i=1, \ldots, 5$.
As discussed in Sec. II we set $\tilde{\xi}_{0}^{2}=\eta^{2} \xi_{0}^{2}, \tilde{\chi}_{a}=\eta^{2} \chi_{a}$, $W=\eta W_{1}$ and rescale time according to Eq. (12). We assume that all faster processes have reached equilibrium. We also expand $M(\boldsymbol{a})=M\left(\boldsymbol{a}_{0}+\eta \boldsymbol{a}_{1}+\eta^{2} \boldsymbol{a}_{2}+O\left(\eta^{3}\right)\right)$ in a Taylor series about the point $\eta=0$ :

$$
\begin{aligned}
M_{i j}\left(\boldsymbol{a}_{0}+\eta \boldsymbol{a}_{1}+\eta^{2} \boldsymbol{a}_{2}+O\left(\eta^{3}\right)\right) \\
=M_{i j}\left(\boldsymbol{a}_{0}\right)+\eta \frac{\partial M_{i j}}{\partial a_{0 k}}\left(\boldsymbol{a}_{0}\right) a_{1 k} \\
\quad+\left(\frac{\partial M_{i j}}{\partial a_{0 k}} a_{2 k}+\frac{1}{2} \frac{\partial^{2} M_{i j}}{\partial a_{0 k} \partial a_{0 l}} a_{1 k} a_{1 l}\right) \eta^{2}+O\left(\eta^{3}\right),
\end{aligned}
$$

where $a_{1 k}$ is the $k$ th component of the vector $\boldsymbol{a}_{1}$. The method of homogenization is as follows: we substitute Eqs. (14) into (A4c) and (A7d) and solve the resulting equations in ascending powers of $\eta$. In doing so we impose appropriate solvability conditions at each order in $\eta$, requiring that all equations have a solution. This requirement imposes constraints on the terms in Eq. (14). Once we have proceeded to $O\left(\eta^{2}\right)$ we will have sufficient constraints to determine the leading-order behavior of the system.

\section{a. The $O$ (1) equations}

Substituting Eqs. (14) into Eqs. (A4c) and (A7d) and retaining terms of order $O(1)$, we obtain

$$
\begin{aligned}
\xi_{0}^{2} \nabla_{\boldsymbol{y}}^{2} \boldsymbol{a}_{0}+M\left(\boldsymbol{a}_{\mathbf{0}}\right) \boldsymbol{a}_{0} & =-\chi_{a} \boldsymbol{e}_{0}, \quad \boldsymbol{y} \in \Omega, \\
\hat{\boldsymbol{n}} \cdot \nabla_{\boldsymbol{y}} \boldsymbol{a}_{0} & =0, \quad \boldsymbol{y} \in \Gamma_{i} .
\end{aligned}
$$

The leading-order electric potential is governed by

$$
\begin{aligned}
\nabla_{\boldsymbol{y}} \cdot\left[\left(\mathcal{I}+\alpha \mathcal{Q}_{0}\right) \nabla_{\boldsymbol{y}} \phi_{0}\right] & =0, \quad \boldsymbol{y} \in \Omega, \\
\phi_{0} & =C_{0 i}, \quad \boldsymbol{y} \in \Gamma_{i}, \\
\int_{\Gamma_{i}}\left[\left(\mathcal{I}+\alpha \mathcal{Q}_{0}\right) \nabla_{\boldsymbol{y}} \phi_{0}\right] \cdot d \boldsymbol{S} & =0, \quad \boldsymbol{y} \in \Gamma_{i} .
\end{aligned}
$$

Notice that $\phi_{0}=\phi_{0}(\boldsymbol{x})$ and $\boldsymbol{a}_{0}=\boldsymbol{a}_{0}(\boldsymbol{x})$ solve Eqs. (A11b) and (A11c) provided that the conditions

$$
\begin{aligned}
M\left(\boldsymbol{a}_{0}\right) \boldsymbol{a}_{0} & =0, \\
\phi_{0} & =C_{0 i}, \quad \boldsymbol{y} \in \Gamma_{i}
\end{aligned}
$$

are satisfied. Condition (A12a) holds for nonzero $\boldsymbol{a}_{0}$ if and only if $\left|a_{0}\right|=S_{0}$ and $\beta_{0}=1-\frac{\left.6 \operatorname{Tr}^{2} \mathcal{Q}_{0}^{3}\right)}{\operatorname{Tr}^{3}\left(\mathcal{Q}_{0}^{2}\right)}=0$ [32]. $S_{0}$ is the leading-order term in the expansion of the scalar order 
parameter and is determined by the temperature $S_{0}=$ $\frac{3+\sqrt{9-8 T_{0}}}{4} . \beta_{0}$ is the biaxiality at leading order. Condition (A12a) fixes $\boldsymbol{a}_{0}$ onto the solution manifold consisting of uniaxial tensors of order parameter $S_{0}$. The remaining degrees of freedom will be determined at higher order in $\eta$. Condition (A12b) constrains the potential to a constant unknown value on the surface of the $i$ th inclusions. If $\phi_{0}$ varies only on the macroscopic scale $\boldsymbol{x}$ there will be a small change in its value, of $O(\eta)$ across a unit cell: as a result condition (A12b) cannot be satisfied and needs to be modified slightly. We Taylor expand $\phi_{0}$ about a point $\boldsymbol{x}_{i}$ on $\Gamma_{i}$ :

$$
\begin{aligned}
\phi_{0}(x)= & \phi_{0}\left(x_{i}\right)+\left(x-x_{i}\right) \cdot \nabla_{x} \phi_{0}\left(x_{i}\right) \\
& +\left(x-x_{i}\right)^{T} \mathcal{H}\left(\phi_{0}\right)\left(x_{i}\right)\left(x-x_{i}\right)+O\left(\left(x-x_{i}\right)^{3}\right),
\end{aligned}
$$

where $\mathcal{H}_{\phi}=\left[\frac{\partial^{2} \phi_{0}}{\partial x_{i} \partial x_{j}}\right]_{\boldsymbol{x}=\boldsymbol{x}_{i}}$. By using the relation $\boldsymbol{x}=\eta \boldsymbol{y}$, we obtain

$$
\begin{aligned}
\phi_{0}(\boldsymbol{x})= & \phi_{0}\left(\boldsymbol{x}_{i}\right)+\eta\left(\boldsymbol{y}-\boldsymbol{y}_{i}\right) \cdot \nabla_{x} \phi_{0}\left(\boldsymbol{x}_{i}\right) \\
& +\eta^{2}\left(\boldsymbol{y}-\boldsymbol{y}_{i}\right)^{T} \mathcal{H}\left(\phi_{0}\right)\left(\boldsymbol{x}_{i}\right)\left(\boldsymbol{y}-\boldsymbol{y}_{i}\right)+O\left(\eta^{3}\right) .
\end{aligned}
$$

Hence we modify the boundary condition (A12b) to

$$
\phi_{0}\left(\boldsymbol{x}_{i}\right)=C_{0 i}, \quad \boldsymbol{y} \in \Gamma_{i} .
$$

Substituting Eqs. (A14) and (14c) into Eq. (A7c) and using Eq. (14f) we obtain an appropriate boundary condition for $\phi_{1}$ :

$$
\phi_{1}=-\left(\boldsymbol{y}-\boldsymbol{y}_{i}\right) \cdot \nabla_{x} \phi_{0}+C_{1 i}, \quad \boldsymbol{y} \in \Gamma_{i} .
$$

The value of the constant $C_{0 i}$ will be fixed at higher order by using the macroscopic boundary conditions.

\section{b. The $O(\eta)$ equations}

At $O(\eta)$, by using $\boldsymbol{a}_{0}=\boldsymbol{a}_{0}(\boldsymbol{x})$ and $\phi_{0}=\phi_{0}(\boldsymbol{x})$ we find

$$
\xi_{0}^{2} \nabla_{y}^{2} \boldsymbol{a}_{1}+M\left(\boldsymbol{a}_{\mathbf{0}}\right) \boldsymbol{a}_{1}+\left(\frac{\partial M}{\partial a_{0 k}}\left(\boldsymbol{a}_{\mathbf{0}}\right) a_{1 k}\right) \boldsymbol{a}_{0}=0, \quad \boldsymbol{y} \in \Omega,
$$

$$
\hat{\boldsymbol{n}} \cdot \nabla_{y} \boldsymbol{a}_{1}+\hat{\boldsymbol{n}} \cdot \nabla_{x} \boldsymbol{a}_{0}=0, \quad \boldsymbol{y} \in \Gamma_{i},
$$

and

$$
\begin{aligned}
& \nabla_{y} \cdot\left[\left(\mathcal{I}+\alpha \mathcal{Q}_{0}\right) \nabla_{y} \phi_{1}\right]=0, \quad y \in \Omega, \\
& \phi_{1}=-\left(y-y_{i}\right) \cdot \nabla_{x} \phi_{0}+C_{1 i}, \quad y \in \Gamma_{i}, \\
& \int_{\Gamma_{i}}\left[\mathcal{I}+\alpha \mathcal{Q}_{0}\right]\left(\nabla_{y} \phi_{1}+\nabla_{x} \phi_{0}\right) \cdot d S=0, \quad y \in \Gamma_{i},
\end{aligned}
$$

where summation over repeated indices is implied. We have simplified Eqs. (A17) and (A18) by using the constraints on $\boldsymbol{a}_{0}$ and $\phi_{0}$ found at $O(1)$. We note that the electric field contribution $\boldsymbol{e}_{1}$, which would appear in Eq. (A17a), is zero because $\phi_{0}$ is independent of $\boldsymbol{y}$. To ensure solvability for $\boldsymbol{a}_{1}$ we project Eq. (A17a) onto the vectors $\boldsymbol{V}^{(k)}$ :

$$
\begin{aligned}
\boldsymbol{V}^{(1)} & =\left[\begin{array}{lllll}
0 & -2 a_{3} & 2 a_{2} & -a_{5} & a_{4}
\end{array}\right], \\
\boldsymbol{V}^{(2)} & =\left[\begin{array}{llllll}
-\sqrt{3} a_{4} & a_{4} & a_{5} & \sqrt{3} a_{1}-a_{2} & -a_{3}
\end{array}\right], \\
\boldsymbol{V}^{(3)} & =\left[\begin{array}{llllll}
\sqrt{3} a_{5} & a_{5} & -a_{4} & a_{3} & -\sqrt{3} a_{1}-a_{2}
\end{array}\right],
\end{aligned}
$$

which span the kernel of the linear operator on $\boldsymbol{a}_{0}$ defined by the left-hand side of Eq. (A10a). In the case of the nematic liquid crystal these vectors have the additional properties of spanning the kernel of the Hessian of the thermotropic part of the free energy density and being tangent to the solution manifold defined by Eq. (A12a) [32]. The solvability condition reads

$$
\int_{\Omega} V_{i}^{(k)}\left(M_{0 i j}+\frac{\partial M_{0 i q}}{\partial a_{0 j}} a_{0 q}\right) a_{1 j} d^{3} y=0
$$

for each vector $\boldsymbol{V}^{(k)}, k=1,2,3$. We recognize the bracketed terms $\mathcal{H}_{i j}=\left(M_{0 i j}+\frac{\partial M_{0 i q}}{\partial a_{0 j}} a_{0 q}\right)$ as the Hessian of the thermotropic free energy. Because the vectors $V^{(k)}$ span its kernel, the solvability condition (A20) is automatically satisfied.

We now proceed to find the solution of Eq. (A17a) subject to Eq. (A17b). We use the ansatz

$$
\boldsymbol{a}_{1}=\sum_{k=1}^{3} \chi_{k} \frac{\partial \boldsymbol{a}_{0}}{\partial x_{k}}+\gamma \boldsymbol{f}\left(\boldsymbol{a}_{0}, \boldsymbol{x}\right)
$$

where we have absorbed the long-scale spatial dependence of $\boldsymbol{a}_{1}$ into the derivative of $\boldsymbol{a}_{0}$ and a general vector valued function $\boldsymbol{f}\left(\boldsymbol{a}_{0}, \boldsymbol{x}\right)$. The vector $\boldsymbol{f}\left(\boldsymbol{a}_{0}, \boldsymbol{x}\right)$ belongs to the same space as $\boldsymbol{a}$ and, as a result, is isomorphic to a traceless symmetric tensor. By writing our ansatz in this form we split the spatial dependence of $\boldsymbol{a}_{1}$ into distinct parts. So that $\chi_{k}$ is uniquely defined we require that $\boldsymbol{f}$ is independent of $\frac{\partial \boldsymbol{a}_{0}}{\partial x_{k}}$ : all terms containing this dependence are contained in the coefficients $\chi_{k}$. Both $\chi_{k}$ and $\gamma$ depend periodically on the microscopic variable $\boldsymbol{y}$ only. The long-scale spatial dependence is contained entirely in the derivative of $\boldsymbol{a}_{0}$ and the function $\boldsymbol{f}\left(\boldsymbol{a}_{0}, \boldsymbol{x}\right)$. Substituting Eq. (A21) into Eqs. (A17a) and (A17b) we obtain two problems. The first is the standard cell problem for elliptic homogenization,

$$
\begin{aligned}
\xi_{0}^{2} \nabla_{y}^{2} \chi_{k} & =0, \quad \boldsymbol{y} \in \Omega, \\
\hat{\boldsymbol{n}} \cdot \nabla_{y} \chi_{k} & =-\hat{\boldsymbol{n}} \cdot \hat{\boldsymbol{e}}_{k}, \quad \boldsymbol{y} \in \Gamma_{i},
\end{aligned}
$$

where we have used the orthogonality of $\boldsymbol{a}_{0}$ with its spatial derivatives to remove the thermotropic term. The second cell problem is

$$
\begin{aligned}
\xi_{0}^{2} \nabla_{\boldsymbol{y}}^{2} \gamma f_{i}+\mathcal{H}_{i k} f_{k} \gamma=0, & \boldsymbol{y} \in \Omega, \\
\hat{\boldsymbol{n}} \cdot \nabla_{\boldsymbol{y}} \boldsymbol{f} \gamma=0, & \boldsymbol{y} \in \Gamma_{i} .
\end{aligned}
$$

We project Eq. (A23a) for $\gamma$ onto $f$ to obtain

$$
\begin{array}{cc}
\nabla_{y}^{2} \gamma+K \gamma=0, & \boldsymbol{y} \in \Omega, \\
\hat{\boldsymbol{n}} \cdot \nabla_{y} \gamma=0, & \boldsymbol{y} \in \Gamma_{i},
\end{array}
$$

where $K=\frac{1}{\xi_{0}^{2} f_{l} f_{l}} f_{i}\left(M_{0 i k}+\frac{\partial M_{0 i j}}{\partial a_{0 k}} a_{0 j}\right) f_{k}$ is the contraction of $f$ with the Hessian $\mathcal{H}$ of the thermotropic free energy and is non-negative because we are at a minimum in the free energy. Hence the only solution to Eq. (A24a) is $\gamma=0$. The remaining cell problem (A22) is solved numerically inside a unit cube with periodic boundary conditions on the outer sides, denoted $\mathcal{B}$ in Fig. 1. A plot of $\chi_{3}$ for a spherical particle of radius $0.4 L_{y}$ is shown in Fig. 6. From this figure we see that $\chi_{k}$ is, up to an unknown constant, modulated in the direction of $\hat{\boldsymbol{e}}_{k}$ with the modulation being strongest near the poles of 


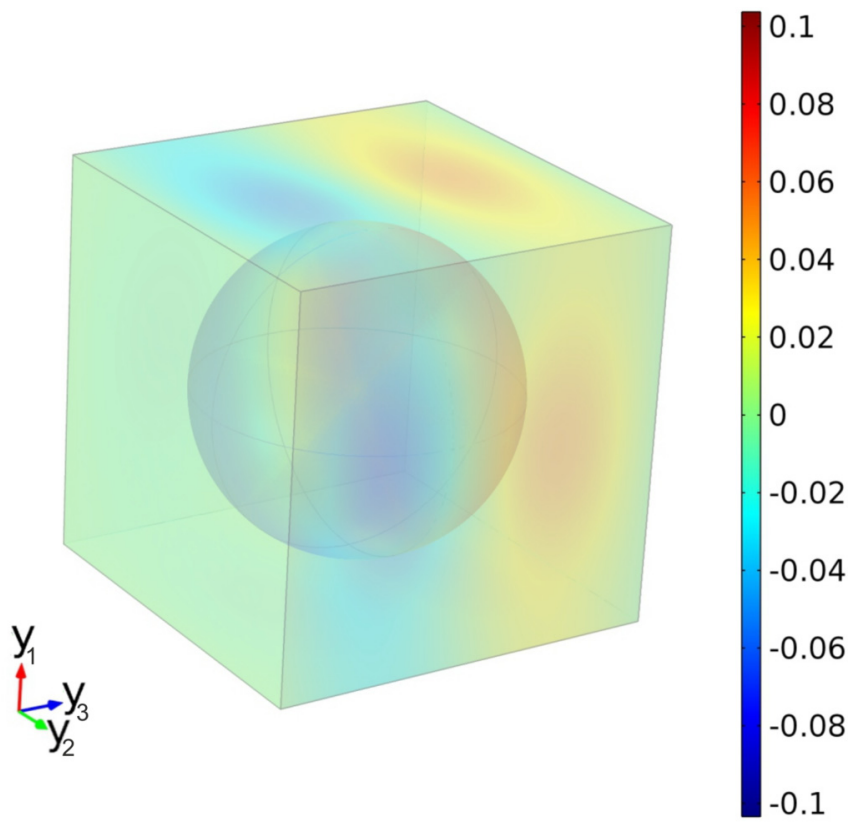

FIG. 6. (Color online) Color-scaled image of the solution $\chi_{3}$ of Eqs. (A22) in the domain around a spherical particle of radius $0.4 L_{y}$. The arbitrary constant has been chosen so that the average modulation of $\chi_{3}$ is zero.

the particle. Because $\chi_{k}$ is not uniquely fixed at $O(\eta)$ we cannot, from this figure alone, determine the behavior of the first-order correction $\boldsymbol{a}_{1}$ in Eq. (A21). However, as already stated in Sec. II C, we do not need to know this correction in order to derive the macroscopic equations for the liquid crystal alignment.

Turning to Eq. (A18), the solvability condition is obtained by integrating Eq. (A18a) over a unit cell and applying the divergence theorem

$$
\int_{\Gamma_{i}}\left(\mathcal{I}+\alpha \mathcal{Q}_{0}\right) \nabla_{y} \phi_{1} \cdot d \boldsymbol{S}=0 .
$$

Substituting Eq. (A18b) into (A25) we find that the solvability condition is satisfied. To obtain the cell problem for $\phi_{1}$ we make the ansatz

$$
\phi_{1}=\sum_{k=1}^{3} R_{k}(\boldsymbol{x}, \boldsymbol{y}) \frac{\partial \phi_{0}}{\partial x_{k}}+\bar{\phi}_{1}(\boldsymbol{x}) .
$$

Substituting Eq. (A26) into Eq. (A18) we find the cell problem

$$
\begin{aligned}
{\left[\delta_{i j}+\alpha \mathcal{Q}_{0 i j}\right] \frac{\partial^{2} R_{k}}{\partial y_{i} \partial y_{j}} } & =0, \quad \boldsymbol{y} \in \Omega, \quad(\mathrm{A} 27 \\
\boldsymbol{R}_{k} & =-\left(y_{k}-y_{0 k}\right), \quad \boldsymbol{y} \in \Gamma_{i},
\end{aligned}
$$

$$
\int_{\Gamma_{i}}\left(\mathcal{I}+\alpha \mathcal{Q}_{0}\right) \nabla_{y} R_{k}(\boldsymbol{y}) \cdot d \boldsymbol{S}=0, \quad \boldsymbol{y} \in \Gamma_{i},
$$

where $k=1,2,3$ and we have set $\bar{\phi}_{1}=C_{1 i}$. The final cell problem $(\mathrm{A} 27 \mathrm{c})$ can be solved numerically subject to periodic boundary conditions on $\mathcal{B}$ (see Fig. 1) once the leading-order alignment tensor $\mathcal{Q}_{0}$ is known. Unlike for Eqs. (A17) a separation of scales is impossible and we cannot obtain a cell problem that is completely independent of the macroscopic variable $\boldsymbol{x}$. While this may appear to be a problem it suffices to solve Eq. (A27c) for a discrete set of $\mathcal{Q}_{0}$ and interpolate.

\section{c. The $O\left(\eta^{2}\right)$ equations}

As a final step we collect terms of $O\left(\eta^{2}\right)$ :

$$
\begin{aligned}
\frac{\partial \boldsymbol{a}_{0}}{\partial t_{2}}= & \xi_{0}^{2}\left(\nabla_{\boldsymbol{y}}^{2} \boldsymbol{a}_{2}+\nabla_{\boldsymbol{x}} \cdot \nabla_{\boldsymbol{y}} \boldsymbol{a}_{1}+\nabla_{\boldsymbol{y}} \cdot \nabla_{\boldsymbol{x}} \boldsymbol{a}_{1}+\nabla_{\boldsymbol{x}}^{2} \boldsymbol{a}_{0}\right) \\
& +M_{0} \boldsymbol{a}_{2}+\left(\frac{\partial M}{\partial a_{0 k}} a_{1 k}\right) \boldsymbol{a}_{1}+\left(\frac{\partial M}{\partial a_{0 k}} a_{2 k}\right) \boldsymbol{a}_{0} \quad(\mathrm{~A} 28 \mathrm{a}) \\
& +\frac{1}{2}\left(\frac{\partial^{2} M}{\partial a_{0 k} \partial a_{0 l}} a_{1 k} a_{1 l}\right) \boldsymbol{a}_{0}+\chi_{a} \boldsymbol{e}_{2}, \quad \boldsymbol{y} \in \Omega, \\
& \hat{\boldsymbol{n}} \cdot \nabla_{\boldsymbol{y}} \boldsymbol{a}_{2}+\hat{\boldsymbol{n}} \cdot \nabla_{\boldsymbol{x}} \boldsymbol{a}_{1}=W_{1}\left(\boldsymbol{a}_{S}-\boldsymbol{a}_{0}\right), \quad \boldsymbol{y} \in \Gamma_{i},
\end{aligned}
$$

(A28b)

$$
\begin{aligned}
0= & \nabla_{\boldsymbol{y}} \cdot\left[\left(\mathcal{I}+\alpha \mathcal{Q}_{0}\right)\left(\nabla_{y} \phi_{2}+\nabla_{x} \phi_{1}\right)+\alpha \mathcal{Q}_{1} \nabla_{y} \phi_{1}\right. \\
& \left.+\alpha \mathcal{Q}_{1} \nabla_{x} \phi_{0}\right]+\nabla_{y} \cdot\left[\alpha \mathcal{Q}_{2} \nabla_{y} \phi_{0}\right]+\nabla_{x} \cdot\left[\left(\mathcal{I}+\alpha \mathcal{Q}_{0}\right)\right. \\
& \left.\times\left(\nabla_{x} \phi_{0}+\nabla_{y} \phi_{1}\right)\right]+\nabla_{x} \cdot\left[\alpha \mathcal{Q}_{1} \nabla_{y} \phi_{0}\right], \quad y \in \Omega,
\end{aligned}
$$

$$
\begin{aligned}
0= & \int_{\Gamma_{i}}\left[\left(\mathcal{I}+\alpha \mathcal{Q}_{0}\right)\left(\nabla_{y} \phi_{2}+\nabla_{x} \phi_{1}\right)\right. \\
& \left.+\alpha \mathcal{Q}_{1}\left(\nabla_{x} \phi_{0}+\nabla_{y} \phi_{1}\right)\right] \cdot d \boldsymbol{S}, \quad y \in \Gamma_{i} .
\end{aligned}
$$

As before we require that Eqs. (A28a) and (A29a) have solutions. We start by projecting Eq. (A28a) onto the kernel of the adjoint of the leading-order operator. The equation governing the alignment of the nematic is

$$
\begin{aligned}
& \int_{\Omega} \boldsymbol{V}^{(k)} \cdot \frac{\partial \boldsymbol{a}_{0}}{\partial t_{2}} d V \\
& =\int_{\Omega} \boldsymbol{V}^{(k)} \cdot\left[\xi_{0}^{2}\left(\nabla_{\boldsymbol{y}}^{2} \boldsymbol{a}_{2}+\nabla_{\boldsymbol{x}} \cdot \nabla_{\boldsymbol{y}} \boldsymbol{a}_{1}+\nabla_{\boldsymbol{y}} \cdot \nabla_{\boldsymbol{x}} \boldsymbol{a}_{1}+\nabla_{\boldsymbol{x}}^{2} \boldsymbol{a}_{0}\right)\right. \\
& \quad+\left(\frac{\partial M}{\partial a_{0 k}} a_{1 k}\right) \boldsymbol{a}_{1}+\mathcal{H} \boldsymbol{a}_{2}+\frac{1}{2}\left(\frac{\partial^{2} M}{\partial a_{0 k} \partial a_{0 l}} a_{1 k} a_{1 l}\right) \boldsymbol{a}_{0} \\
& \left.\quad+\xi_{0}^{2} \chi_{a} \boldsymbol{e}_{2}\right] d V .
\end{aligned}
$$

Differentiating Eq. (A5) we find

$$
\frac{\partial^{2} M_{i j}}{\partial a_{0 k} \partial a_{0 l}}=-4 \delta_{i j} \delta_{k l},
$$

hence $\boldsymbol{V}^{(k)} \cdot \frac{1}{2}\left(\frac{\partial^{2} M}{\partial a_{0 k} \partial a_{0 l}} a_{1 k} a_{1 l}\right) \boldsymbol{a}_{0}$ is zero. It can be shown with some algebra that $\frac{\partial M}{\partial a_{0 k}} a_{1 k} \boldsymbol{a}_{1}$ is also zero for a uniaxial nematic. We use Eq. (A8c) to write

$$
\begin{aligned}
\boldsymbol{V}^{(k)} \cdot \int_{\Omega} \boldsymbol{e}_{2} d V= & \sum_{i=1}^{5} V_{i}^{(k)} \operatorname{Tr}\left[\nabla_{\boldsymbol{x}} \phi_{0} \otimes \nabla_{\boldsymbol{x}} \phi_{0}\left(|\Omega|+2\left|\Omega_{n p}\right|\right) \mathcal{T}^{(i)}\right. \\
& \left.+\int_{\Omega} \nabla_{\boldsymbol{y}} \phi_{1} \otimes \nabla_{\boldsymbol{y}} \phi_{1} d V \mathcal{T}^{(i)}\right],
\end{aligned}
$$


where $|\Omega|$ is the volume of liquid crystal contained in a unit cell and $\left|\Omega_{n p}\right|$ is the volume of an inclusion and we used

$$
\int_{\Omega} \nabla_{\boldsymbol{y}} \phi_{1} d^{3} y=\sum_{k=1}^{3} \hat{\boldsymbol{e}}_{k} \cdot \nabla_{\boldsymbol{x}} \phi_{0}(\boldsymbol{x}) \int_{\Omega} \nabla_{\boldsymbol{y}} R_{k}(\boldsymbol{y}) d^{3} y,
$$

the divergence theorem, and the boundary condition (A27b) to obtain

$$
\int_{\Omega} \nabla_{\boldsymbol{y}} R_{k}(\boldsymbol{y}) d^{3} y=\int_{\Gamma_{i}} R_{k}(\boldsymbol{y}) \hat{\boldsymbol{n}} d S=\left|\Omega_{n p}\right| \hat{\boldsymbol{e}}_{k} .
$$

Finally, by using Eqs. (A31) and (A32) and the fact that $\boldsymbol{V}^{(k)}$ span the kernel of the Hessian $\mathcal{H}$, Eq. (A30) reduces to

$$
\begin{aligned}
\boldsymbol{V}^{(k)} \cdot \frac{\partial \boldsymbol{a}_{0}}{\partial t_{2}}= & \boldsymbol{V}^{(k)} \cdot\left\{\xi_{0}^{2} \nabla_{\boldsymbol{x}} \cdot\left(D \nabla_{\boldsymbol{x}} \boldsymbol{a}_{0}\right)\right. \\
& \left.+\chi_{a}\left[\boldsymbol{e}_{M}\left(1+2 \frac{\left|\Omega_{n p}\right|}{|\Omega|}\right)+\boldsymbol{p}\right]+W_{1} \xi_{0}^{2} \boldsymbol{q}\right\}
\end{aligned}
$$

for $k=1,2,3$, with the constraint that $\left|\boldsymbol{a}_{0}\right|^{2}=S_{0}^{2}$ and that the leading-order biaxiality is zero. Here,

$$
\begin{gathered}
\mathcal{D}_{i j}=\frac{1}{|\Omega|} \int_{\Omega}\left(\delta_{i j}+\frac{\partial \chi_{j}}{\partial y_{i}}\right) d^{3} y, \\
\boldsymbol{q}=\frac{1}{|\Omega|} \int_{\Gamma} \boldsymbol{a}_{S} d S,
\end{gathered}
$$

and the components of the macroscopic electric field $\boldsymbol{e}_{M}$ and polarization field $\boldsymbol{p}$ are

$$
\begin{gathered}
e_{M i}=\operatorname{Tr}\left[\nabla_{x} \phi_{0} \otimes \nabla_{x} \phi_{0} \mathcal{T}^{(i)}\right], \\
p_{i}=\frac{1}{|\Omega|} \int_{\Omega} \operatorname{Tr}\left[\nabla_{y} \phi_{1} \otimes \nabla_{y} \phi_{1} \mathcal{T}^{(i)}\right] d V .
\end{gathered}
$$

When supplemented with the macroscopic boundary conditions, Eqs. (A35)-(A37) form a complete macroscopic description of the liquid crystal alignment.

The solvability condition for the the electric field is

$$
\begin{aligned}
0= & |\Omega| \nabla_{\boldsymbol{x}} \cdot\left[\left(\mathcal{I}+\alpha \mathcal{Q}_{0}\right) \nabla_{\boldsymbol{x}} \phi_{0}\right] \\
& +\nabla_{\boldsymbol{x}} \cdot\left[\left(\mathcal{I}+\alpha \mathcal{Q}_{0}\right) \int_{\Omega} \nabla_{\boldsymbol{y}} \phi_{1} d^{3} y\right] .
\end{aligned}
$$

By using Eq. (A34) we find that the homogenized equation for the electric potential is

$$
\nabla_{x} \cdot\left[\left(|\Omega|+\left|\Omega_{n p}\right|\right)\left(\mathcal{I}+\alpha \mathcal{Q}_{0}\right) \nabla_{x} \phi_{0}\right]=0 .
$$

We supplement this expression with the macroscopic boundary conditions (A7b). Because the factor $|\Omega|+\left|\Omega_{n p}\right|$ is a constant, the presence of uniformly distributed identical metallic inclusions has no effect on equations governing the leading-order potential.
[1] C. P. Lapointe, T. G. Mason, and I. I. Smalyukh, Science 326, 1083 (2009).

[2] A. Martinez, M. Ravnik, B. Lucero, R. Visvanathan, S. Žumer, and I. I. Smalyukh, Nat. Mater. 13, 258 (2014).

[3] S. Čopar and S. Žumer, Phys. Rev. Lett. 106, 177801 (2011).

[4] T. Araki and H. Tanaka, Phys. Rev. Lett. 97, 127801 (2006).

[5] I. Muševič, M. Škarabot, U. Tkalec, M. Ravnik, and S. Žumer, Science 313, 954 (2006).

[6] H. Stark, Eur. Phys. J. B 10, 311 (1999).

[7] R. W. Ruhwandl and E. M. Terentjev, Phys. Rev. E 56, 5561 (1997).

[8] T. Hegmann, H. Qi, B. Kinkead, V. Marx, H. Girgis, and P. Heiney, Can. Metall. Q. 48, 1 (2009).

[9] S. K. Prasad, K. L. Sandhya, G. G. Nair, U. S. Hiremath, C. V. Yelamaggad, and S. Sampath, Liq. Cryst. 33, 1121 (2006).

[10] T. Miyama, J. Thisayukta, H. Shiraki, Y. Sakai, Y. Shiraishi, N. Toshima, and S. Kobayashi, Jpn. J. Appl. Phys., Part 1 43, 2580 (2004).

[11] H. Qi, B. Kinkead, and T. Hegmann, Adv. Funct. Mater. 18, 212 (2008).

[12] R. Pratibha, K. Park, I. I. Smalyukh, and W. Park, Opt. Express 17, 19459 (2009).

[13] B. Senyuk, J. S. Evans, P. J. Ackerman, T. Lee, P. Manna, L. Vigderman, E. R. Zubarev, J. v. d. Lagemaat, and I. I. Smalyukh, Nano Lett. 12, 955 (2012).

[14] O. Buchnev, J. Y. Ou, M. Kaczmarek, N. I. Zheludev, and V. A. Fedotov, Opt. Express 21, 1633 (2013).

[15] F. Brochard and P. de Gennes, J. Phys. (Paris) 31, 691 (1970).
[16] S. V. Burylov and Y. L. Raikher, Mol. Cryst. Liq. Cryst. Sci. Technol., Sect. A 258, 107 (1995).

[17] S. V. Burylov and Y. L. Raikher, Mol. Cryst. Liq. Cryst. Sci. Technol., Sect. A 258, 123 (1995).

[18] S. V. Burylov, V. I. Zadorozhnii, I. P. Pinkevich, V. Y. Reshetnyak, and T. J. Sluckin, Mol. Cryst. Liq. Cryst. 375, 525 (2002).

[19] V. Y. Reshetnyak, S. M. Shelestiuk, and T. J. Sluckin, Mol. Cryst. Liq. Cryst. 454, 201 (2006).

[20] S. M. Shelestiuk, V. Y. Reshetnyak, and T. J. Sluckin, Phys. Rev. E 83, 041705 (2011).

[21] L. M. Lopatina and J. V. Selinger, Phys. Rev. Lett. 102, 197802 (2009).

[22] L. M. Lopatina and J. V. Selinger, Phys. Rev. E 84, 041703 (2011).

[23] N. Podoliak, O. Buchnev, O. Buluy, G. D'Alessandro, M. Kaczmarek, Y. Reznikov, and T. J. Sluckin, Soft Matter 7, 4742 (2011).

[24] G. Pavliotis and A. Stuart, Multiscale Methods: Averaging and Homogenization, 1st ed. (Springer-Verlag, New York, 2007).

[25] Q. Shen and M. C. Calderer, Math. Methods Appl. Sci. 27, 1125 (2004).

[26] L. Berlyand, D. Cioranescu, and D. Golovaty, J. Math. Pure Appl. 84, 97 (2005).

[27] L. Berlyand and E. Khruslov, J. Math. Phys. 46, 095107 (2005).

[28] P. G. de Gennes, C. R. Acad. Sci. Paris B 275, 319 (1972).

[29] A. Sonnet, A. Kilian, and S. Hess, Phys. Rev. E 52, 718 (1995). 
[30] O. V. Kuksenok, R. W. Ruhwandl, S. V. Shiyanovskii, and E. M. Terentjev, Phys. Rev. E 54, 5198 (1996).

[31] A. Sonnet, P. Maffettone, and E. Virga, J. Non-Newtonian Fluid Mech. 119, 51 (2004).

[32] K. R. Daly, G. D’Alessandro, and M. Kaczmarek, SIAM J. Appl. Math. 70, 2844 (2010).

[33] I. W. Stewart, The Static and Dynamic Continuum Theory of Liquid Crystals: A Mathematical Introduction (Taylor \& Francis, London \& New York, 2004).
[34] L. N. Trefethen, Spectral Methods in Matlab (SIAM, Philadelphia, 2000).

[35] W. Haiss, N. T. K. Thanh, J. Aveyard, and D. G. Fernig, Anal. Chem. 79, 4215 (2007).

[36] U. Hornung, Homogenization and Porous Media, edited by L. S. L. Kadanoff, J. E. Marsden, and S. Wiggins (Springer, Berlin, 1996).

[37] O. Iliev, A. Mikelic, and P. Popov, Multiscale Model. Simul. 7, 93 (2008). 